\title{
EXTENDING HOMEOMORPHISMS ON THE PSEUDO-ARC
}

BY

\author{
G. R. LEHNER(1)
}

1. Introduction. Appearing simultaneously with the definition of the term homogeneous by Sierpinski [7] was the following question proposed by Knaster and Kuratowski [4]: Is the simple closed curve the only nondegenerate, bounded, homogeneous plane continuum? Partial results were obtained by Mazurkiewicz [5] and Cohen [3] and incorrect solutions were presented by Waraskiewicz [8] and Choquet [2]. In 1948 Bing [1] settled the question by proving that the pseudo-arc was homogeneous.

This paper is the result of an effort to extend this result in a direction suggested by the following question. If $H$ is a subset of the pseudo-arc $M$ and $T$ is a homeomorphism of $H$ onto a subset $T(H)$ of $M$, what conditions will imply the existence of an extension of $T$ to a homeomorphism of $M$ onto $M$ ? (Note that in case $H$ is a single point, a condition is that $M$ be homogeneous.) The main result of this paper answers this question in case $H$ is closed and contains at most a finite number of components.

In particular, we obtain the following:

Theorem 6. Suppose $H_{1,1}, H_{1,2}, \cdots, H_{1 n}$, are disjoint subcontinua of the pseudo-arc $M$ and that $M$ is irreducible between each pair of them. Suppose $T$ is a homeomorphism of $H_{1,1}+H_{1,2}+\cdots+H_{1, n}$ onto $H_{2,1}+H_{2,2}+\cdots+H_{2, n}$ where $H_{2,1}, H_{2,2}, \cdots, H_{2, n}$ are subcontinua of $M$ such that $M$ is irreducible between each pair of them. Then $T$ can be extended to a homeomorphism of $M$ onto $M$.

Theorem 7. Suppose $\mathfrak{H}_{1}, \mathfrak{H}_{2}$ are closed subsets of the pseudo-arc $M$ and each have the same finite number of components $H_{1,1}, H_{1,2}, \cdots, H_{1, n}$ and $H_{2,1}$, $H_{2,2}, \cdots, H_{2, n}$ respectively. Suppose also that $T$ is a homeomorphism of $\mathfrak{H C}_{1}$ onto $\mathcal{F}_{2}$. Let $m$ be the maximum of 2 and $n-1$. Then a necessary and sufficient condition that $T$ can be extended to a homeomorphism of $M$ onto $M$ is that for any $m$ points $P_{1}, P_{2}, \cdots, P_{m}$ of $\mathcal{F}_{1}$, there exists a homeomorphism of $M$ onto $M$ taking $P_{i}$ onto $T\left(P_{i}\right)(i=1,2, \cdots, m)$.

2. Some definitions and notation. A set $M$ is homogeneous if, for each pair of its points $P$ and $Q$, there exists a homeomorphism of $M$ onto itself that carries $P$ onto $Q$.

Presented to the Society, April 18, 1958; received by the editors July 28, 1959.

(1) This paper is part of the author's doctoral thesis at the University of Wisconsin. The author is indebted to Professor R. H. Bing, who directed the progress of this work.

The preparation of this paper was supported in part by the National Science Foundation. 
A chain $D=\left[d_{1}, d_{2}, \cdots, d_{m}\right]$ is a finite collection of open sets $d_{1}, d_{2}, \cdots$, $d_{m}$ such that $d_{i}$ intersects $d_{j}$ if $|i-j| \leqq 1$ and the closure of $d_{i}$ intersects the closure of $d_{j}$ only if $|i-j| \leqq 1 . d_{i}$ is called the $i$ th link of $D ; d_{1}$ and $d_{m}$ are the end links of $D$ while links that are not end links are interior links. Two links are said to be adjacent if they intersect. If $P$ and $Q$ are points that belong to $d_{1}$ and $d_{m}$ respectively but to no other links, $D$ is a chain from $P$ to $Q$.

A chain $D$ contains a chain $E$ if each link of $E$ is a subset of a link of $D$. A chain $D$ is a consolidation of a chain $E$ if each link of $D$ is the sum of links of $E$ and $D$ contains $E$.

A chain $E$ each of whose links is a link of $D$ is termed a subchain of $D$. $D(i, j)$ denotes the subchain of $D$ whose end links are $d_{i}$ and $d_{j}$. If $P$ and $Q$ are points each of which lies in exactly one link of $D$, then $d_{P}$ and $D_{Q}$ denote these links and $D(P, Q)$ is the subchain of $D$ with end links $d_{P}$ and $d_{Q} . d_{P}$ $<d_{Q}$ means $d_{P}$ precedes $d_{Q}$ in the chain $D$.

$D^{*}$ denotes the sum of the elements of $D$. The mesh of $D$ (denoted by $\mu(D))$ is the maximum of the diameters of the links of $D$. If the mesh of $D$ is less than $\epsilon$, then $D$ is an $\epsilon$-chain. If $D$ covers a space $M$, the Lebesgue number of $D$ is the least upper bound of all numbers $\delta$ such that any subset of $M$ of diameter less than $\delta$ is contained in a link of $D$.

A chain $E=\left[e_{1}, e_{2}, \cdots, e_{n}\right]$ is crooked in a chain $D=\left[d_{1}, d_{2}, \cdots, d_{m}\right]$ if $D$ contains $E$ and whenever $e_{h}, e_{k}$, intersect $d_{r}, d_{s}$ respectively with $|r-s|$ $>2$, then $E(h, k)$ is the sum of three subchains $E(h, i), E(i, j)$ and $E(j, k)$ such that $(i-j)(h-k)$ is positive and $e_{i}, e_{j}$ are subsets of the links of $D(r, s)$ adjacent to $d_{s}, d_{r}$ respectively.

If $\left(x_{1}, y_{1}\right),\left(x_{2}, Y_{2}\right), \cdots,\left(x_{n}, y_{n}\right)$ is a collection of ordered pairs of positive integers, the chain $E$ follows the pattern $\left(x_{1}, y_{1}\right),\left(x_{2}, y_{2}\right), \cdots,\left(x_{n}, y_{n}\right)$ in the chain $D$ provided that the $x_{i}$ th link of $E$ is a subset of the $y_{i}$ th link of $D(i=1,2, \cdots, n)$. Note that $E$ may follow more than one pattern in $D$.

3. Some properties of crooked chains. The results of this section were originally proved by Bing [1]. However, the proof of Theorem 1 (below) as given there has presented some readers with difficulty and it is at the request of Professor Bing that a more detailed version is presented here. For the proofs of Lemmas 1 and 2 the reader is referred to [1].

Lemma 1. If $D, E$, and $F$ are chains such that $D$ is a consolidation of $E$ and $F$ is crooked in $E, F$ is crooked in $D$.

Lemma 2. If $D, E$, and $F$ are chains such that $F$ is contained in $E$ and $E$ is crooked in $D, F$ is crooked in $D$.

THEOREM 1. Suppose $x_{1}, x_{2}, \cdots, x_{n}$ is a collection of positive integers such that $h=x_{1} \leqq x_{i} \leqq x_{n}=k$ and $\left|x_{i}-x_{i+1}\right| \leqq 1 \quad(i=1,2, \cdots, n-1)$. Suppose also that $D_{1}, D_{2}, \cdots$ is a sequence of chains from $P$ to $Q$ such that for each positive integer $i, D_{i+1}$ is crooked in $D_{i}$, the closure of each link of $D_{i+1}$ is a compact sub- 
set of a link of $D_{i}$, and the mesh of $D_{i}$ is less than $1 / i$. Let $d(i)_{r}$ denote the rth link of $D_{i}$. Suppose further that the subchain $D_{2}(u, v)$ of $D_{2}$ is contained in the subchain $D_{1}(h, k)$ of $D_{1}$ and the closures of $d(2)_{u}$ and $d(2)_{v}$ are mutually exclusive subsets of $d(1)_{h}$ and $d(1)_{k}$ respectively. Then for each integer w there is an integer $j$ greater than $w$ and a chain $E=\left[e_{1}, e_{2}, \cdots, e_{n}\right]$ following the pattern $\left(1, x_{1}\right),\left(2, x_{2}\right), \cdots,\left(n, x_{n}\right)$ in $D_{1}$ such that $E$ is a consolidation of the links of $D_{j}$ contained in $D_{2}(u, v)$ and no interior link of $E$ intersects $d(2)_{u}+d(2)_{v}$.

Proof. The proof will be given by an induction on $n$ and considering four cases. Let $w$ be any integer. Since $\left|x_{i}-x_{i+1}\right| \leqq 1, n$ is at least as great as $k-h+1$.

CASE 1. Suppose $n=k-h+1$. Since the closures of $d(2)_{u}$ and $d(2)_{v}$ are mutually exclusive compact subsets of $d(1)_{h}$ and $d(1)_{k}$ respectively, there exists an integer $j>w$ such that any link of $D_{j}$ that intersects $d(2)_{u}$ or $d(2)_{v}$ is contained in $d(1)_{h}$ or $d(1)_{k}$ respectively and no link of $D_{j}$ intersects both $d(2)_{u}$ and $d(2)_{v}$. Now let $e_{1}$ be the sum of the links of $D_{j}$ that are contained in the intersection of $D_{2}^{*}(u, v)$ and $d(1)_{h}$. Let $e_{n}$ (the last link of $E$ ) be the sum of the links of $D_{j}$ that are contained in the intersection of $D_{2}^{*}(u, v)$ and $d(1)_{k}$. Let $e_{i}(i=2,3, \cdots, n-1)$ be the sum of the remaining links of $D_{j}$ that are contained in the intersection of $D_{2}^{*}(u, v)$ and $d(1)_{x_{i}}$. This proves the theorem for the case $n=k-h+1$.

Now suppose $k, h, n$ are fixed positive integers such that $n>k-h+1$ and that the theorem holds for all positive integers less than $n$. We shall prove that it holds for this value of $n$.

CASE 2. Consider first the situation where $x_{1}=x_{2}$. By the induction hypothesis, there exists a positive integer $k>w$ and a chain $F=\left[f_{1}, f_{2}, \cdots, f_{n-1}\right]$ such that $F$ is a consolidation of the links of $D_{k}$ in $D(u, v)$, only the first link of $F$ intersects $d(2)_{u}$, only the last link of $F$ intersects $d(2)_{v}$, and $F$ follows the pattern $\left(1, x_{2}\right),\left(2, x_{3}\right), \cdots,\left(n-1, x_{n}\right)$ in $D_{1}$.

Let $j$ be an integer greater than $k$ and such that there exists a link of $D_{j}$ that is contained in $f_{1}$ but does not intersect $d(2)_{u}$.

Define $E=\left[e_{1}, e_{2}, \cdots, e_{n}\right]$ as follows. $e_{1}$ is the sum of all those links of $D_{j}$ that are contained in $f_{1}$ and intersect $d(2)_{u}$ plus those links of $D_{j}$ that are contained in $d(2)_{u}$ but are not contained in any link of $F . e_{2}$ is the sum of all those links of $D_{j}$ that are contained in $f_{1}$ but that are not contained in $e_{1}$. In general, $e_{i}$ is the sum of all those links of $D_{j}$ that are contained in $f_{i-1}$ but that are not contained in $e_{1}+e_{2}+\cdots+e_{i-1}(i=2,3, \cdots, n-1)$. Finally, $e_{n}$ is the sum of all those links of $D_{j}$ that are contained in $f_{n-1}+d(2)_{v}$ but that are not contained in $e_{1}+e_{2}+\cdots+e_{n-1}$.

CASE 3. Now we consider the case where $x_{1} \neq x_{2}$ but where there exists an integer $r \neq 2$ such that $x_{1}=x_{r}$. Then there also exists an integer $t$ with $1<t<r<n$ such that $x_{r}<x_{t}$ and $x_{i}<x_{t}(i=1,2, \cdots, r)$.

By hypothesis, the closure of each link of $D_{2}$ is a compact subset of a 
link of $D_{1}$ and in particular the closures of $d(2)_{u}, d(2)_{v}$, are mutually exclusive subsets of $d(1)_{h}, d(1)_{k}$ respectively. Since $\mu\left(D_{i}\right)<1 / i$, there exists an integer $m>w$ such that each five-linked subchain of $D_{m}$ that is contained in $D_{2}(u, v)$ is such that the closure of the sum of the links of this subchain is contained in a link of $D_{1}(h, k)$, any five-linked subchain of $D_{m}$ one of whose links intersects $d(2)_{u}$ or $d(2)_{v}$ is contained in $d(1)_{k}$ or $d(1)_{k}$ respectively, and the sum of no four consecutive links of $D_{m}$ intersects $d(2)_{u}$ and $d(2)_{v}$.

Now suppose $d(2)_{v}$ is not contained in $D_{1}\left(h, x_{t}\right)$. Then there exists a subchain of $D_{m+1}$ that is contained in $D_{2}(u, v)$ and whose end links intersect $d(2)_{u}$ and $d(1)_{x_{t}+1}$ respectively. Let $D_{m+1}(p, q)$ be such a subchain of $D_{m+1}$ that satisfies the additional condition that no interior link of $D_{m+1}(p, q)$ intersects $d(2)_{u}$ or $d(1)_{x_{t}+1}$. Because of the way $m$ was chosen and since $D_{m+1}$ is crooked in $D_{m}$, it is true that $D_{m+1}(p, q)$ is the sum of three chains $D_{m+1}(p, y)$, $D_{m+1}(y, z), D_{m+1}(z, q)$ such that $(q-p)(z-y)$ is positive, the closure of $d(m+1)_{y}$ is contained in $d(1)_{x_{t}}$, and the closure of $d(m+1)_{z}$ is contained in $d(1)_{h}$. Furthermore since no interior link of $D_{m+1}(p, q)$ intersects $d(1)_{x_{t}+1}$, $D_{m+1}(p, z)$ is contained in $D_{1}\left(h, x_{t}\right)$ and no link of $D_{m+1}(p, z)$ intersects $d(2)_{\text {。 }}$.

On the other hand, suppose $d(2)_{v}$ is contained in $d(1)_{x_{i}}$. Then there exists a subchain $D_{m+1}(p, q)$ of $D_{m+1}$ that is contained in $D_{2}(u, v)$ whose end links intersect $d(2)_{u}, d(2)_{v}$ respectively, and none of whose interior links intersect $d(2)_{u}$ or $d(2)_{v}$. Again because of the way $m$ was chosen and since $D_{m+1}$ is crooked in $D_{m}$, we find that $D_{m+1}(p, q)$ is the sum of three chains $D_{m+1}(p, y)$, $D_{m+1}(y, z), D_{m+1}(z, q)$ satisfying the same conditions as in the preceding paragraph. These properties of $D_{m+1}(p, q)$ will be used in what follows in order to be sure that certain sets are non-null.

We now divide the links of $D_{m+1}$ that are contained in $D_{2}(u, v)$ into a number of classes as follows.

$A_{1}$ is the set of all links that intersect $d(2)_{u} . A_{2}$ is the set of all links whose closures lie in $d(1)_{x_{t}}$ but which do not intersect $d(2)_{v} .\left(d(m+1)_{y}\right.$ and $d(m+1)_{q-1}$ belong to $A_{2}$.) $A_{3}$ is the set of all links of subchains of $D_{m+1}$ that are irreducible with respect to having one end link in $A_{1}$ and another in $A_{1}+A_{2}$. (Note that the links of $D_{m+1}(p, y)$ are in $A_{3}$.)

$B_{1}$ is the set of all links that are not in $A_{1}+A_{2}+A_{3}$ but whose closures are in $d(1)_{h} .\left(d(m+1)_{2}\right.$ is in $B_{1}$.) Note that $A_{1}+A_{2}+A_{3}$ and $B_{1}$ are disjoint $B_{2}$ is the set of all links of subchains of $D_{m+1}$ that are irreducible with respect to having one end link in $B_{1}$ and another in $A_{2}$. (Note that the links of $D_{m+1}(z, q-1)$ are in $B_{2}$.)

Let $C_{1}$ be the sum of the remaining links of $D_{m+1}$.

We shall now construct three chains $A, B, C$ using these sets.

The chain $A$ is defined as follows. The first link of $A$ is the sum of the links in $A_{1}$ together with those links that belong to $A_{3}$ but not to $A_{2}$ and whose closures are contained in $d(1)_{x_{1}}$. The $i$ th link of $A$ is the sum of those links that belong to $A_{3}$ but to neither $A_{1}$ nor $A_{2}$ and whose closures are contained 
in $d(1)_{x_{1}+(i-1)}\left(i=2,3, \cdots, x_{t}-x_{1}\right)$. The last link of $A$ is the sum of those links that belong to $A_{2}$ or $A_{3}$ but not $A_{1}$ and whose closures are contained in $d(1)_{x_{i}}$. Then $A$ is a chain that is contained in $D_{1}\left(x_{1}, x_{t}\right)$ and the closures of its first and last links are disjoint subsets of $d(1)_{x_{1}}$ and $d(1)_{x_{t}}$ respectively.

The chain $B$ is defined in a similar way using the elements of $B_{1}, B_{2}$, and $A_{2}$ so as to obtain a chain $B$ that is contained in $D\left(x_{1}, x_{t}\right)$, whose first and last links contain $B_{1}^{*}$ and $A_{2}^{*}$ respectively, and the closures of these first and last links are mutually exclusive subsets of $d(1)_{x_{1}}$ and $d(1)_{x_{t}}$ respectively. In addition, only the last links of $A$ and $B$ intersect.

Finally, the links in $B_{1}$ and $C_{1}$ are added together to obtain a chain $C$ as follows. The first link of $C$ is the sum of the links of $B_{1}$ plus the links in $C_{1}$ whose closures are contained in $d(1)_{x_{1}}$. The $i$ th link of $C$ is the sum of the links in $C_{1}$ whose closures are contained in $d(1)_{x_{1}+(i-1)}\left(i=2,3, \cdots, x_{n}-x_{1}+1\right)$. Then the closures of the first and last links of $C$ are disjoint subsets of $d(1)_{x_{1}}$ and $d(1)_{x_{n}}$ respectively, only the last link of $C$ intersects $d(2)_{v}$, only the first links of $B$ and $C$ intersect, and $A^{*}$ and $C^{*}$ are disjoint.

By making use of the induction hypothesis we can find an integer $j_{1}$ greater than $m$ and a chain $F$ such that $F$ is a consolidation of the links of $D_{j_{1}}$ that are contained in $A_{3}$, only the first and last links of $F$ contain points of $A_{1}^{*}$ and $A_{2}^{*}$ respectively, and $F$ follows the pattern $\left(1, x_{1}\right),\left(2, x_{2}\right), \cdots,\left(t, x_{t}\right)$ in $D_{1}$. For the same reason, there exists an integer $j_{2}$ greater than $m$ and a chain $G$ such that $G$ is a consolidation of the links of $D_{j_{2}}$ that are contained in $B$, only the first and last links of $G$ contain points of $A_{2}^{*}$ and $B_{1}^{*}$ respectively, and $G$ follows the pattern $\left(1, x_{t}\right),\left(2, x_{t+1}\right), \cdots,\left(r+1-t, x_{r}\right)$ in $D_{1}$. Similarly, there exists an integer $j_{3}$ greater than $m$ and a chain $H$ such that $H$ is a consolidation of the links of $D_{j_{2}}$ that are contained in $C$, only the first and last links of $H$ contain points of $B_{1}^{*}$ and $d(2)$, respectively, and $H$ follows the pattern $\left(1, x_{r}\right),\left(2, x_{r+1}\right), \cdots,\left(n-1-r, x_{n}\right)$ in $D_{1}$.

Let $j$ be the maximum of $j_{1}, j_{2}, j_{3}$. Then we can define the desired chain $E$ as follows. $E$ will be a consolidation of the links of $D_{j}$ that are contained in $D_{2}(u, v)$ where $e_{1}$ is the sum of those links of $D_{j}$ in $D_{2}(u, v)$ that are contained in either $d(2)_{u}$ or the first link of $F ; e_{i}$ is the sum of the links of $D_{j}$ in $D_{2}(u, v)$ that are contained in the $i$ th link of $F(i=2,3, \cdots, t-1) ; e_{t}$ is the sum of the links of $D_{j}$ in $D_{2}(u, v)$ that are contained in the last link of $F$ or the first link of $G$; the next $(r+1-t)-2$ links of $E$ are the sum of the links of $D_{j}$ in $D_{2}(u, v)$ that are contained in some particular interior link of $G$; the next link of $E$ is the sum of the links of $D_{j}$ in $D_{2}(u, v)$ that are contained in the last link of $G$ or the first link of $H$; the next $(n-1-r)-2$ links of $E$ are the sum of the links of $D_{j}$ in $D_{2}(u, v)$ that are contained in some particular interior link of $H$; and the last link of $E$ is the sum of the links of $D_{j}$ in $D_{2}(u, v)$ that are subsets of $d(2)_{v}$ or the last link of $H$. This gives us the desired chain $E$ if for some integer $i$ greater than $1, x_{i}=x_{1}$.

CASE 4 . Now assume that $x_{i} \neq x_{1}(i=2,3, \cdots, n)$. By the symmetry of 
the preceding arguments, we can also assume that $x_{i} \neq x_{n}(i=1,2, \cdots, n-1)$. Hence if $x_{n}-x_{1}=1, n$ can only equal 2 and then the theorem follows by Case 1 .

Two possibilities remain: $x_{n}-x_{1}=2$ and $x_{n}-x_{1}>2$. We shall consider this latter case first.

Let $U$ be the set of all links of $D_{2}(u, v)$ that are not contained in $d(1)_{x_{1}}$. Then we define a chain $F=\left[f_{1}, f_{2}, \cdots, f_{x_{n}-x_{1}}\right]$ as follows. $f_{i}$ is the sum of those elements of $U$ whose closures are contained in

$$
d(1)_{x_{1}+i} \quad\left(i=12 \ldots x_{n}-2 x_{1}\right) .
$$

Since $x_{n}-x_{1}>2$, the closures of $f_{1}$ and $f_{x_{n}-x_{1}}$ are disjoint subsets of $d(1)_{x_{1}+1}$ and $d(1)_{x_{n}}$ respectively and $d(2)_{v}$ is contained in $f_{x_{n}-x_{1}}$.

By the induction hypothesis, there is an integer $j$ greater than $m$ (as defined in Case 3 ) and a chain $G=\left[g_{1}, g_{2}, \cdots, g_{n-1}\right]$ such that $G$ is a consolidation of the links of $D_{j}$ that are contained in $F^{*}$, only $g_{1}$ intersects $f_{1}$, only $g_{n-1}$ intersects $f_{x_{n}-x_{1}}$, and $G$ follows the pattern $\left(1, x_{2}\right),\left(2, x_{3}\right), \cdots$, $\left(n-1, x_{n}\right)$ in $D_{1}$. (Note that since only $g_{n-1}$ intersects $f_{x_{n}-x_{1}}$, only $g_{n-1}$ intersects $d(2)_{v}$.) Now we define the desired chain $E$ as follows: $e_{1}$ is the sum of all the links of $D_{j}$ in the intersection of $D_{2}^{*}(u, v)$ and $d(1)_{x_{1}}$ and $e_{i+1}=g_{i}$ $(i=1,2, \cdots, n-1)$.

Now suppose $x_{n}-x_{1}=2$. Then $d(1)_{x_{1}+1}=d(1)_{x_{2}}=\cdots=d(1)_{x_{n-1}}=d(1)_{x_{n}-1}$. Let $F=\left[f_{1}, f_{2}, f_{3}\right]$ be a chain defined as follows: $f_{i}$ is the sum of the links of $D_{2}(u, v)$ whose closures are contained in $d(1)_{x_{1}+(i-1)}(i=1,2,3)$. Note that the closures of $f_{1}, f_{3}$ are disjoint and contain $d(2)_{u}, d(2)_{v}$ respectively. Since $\mu\left(D_{i}\right)<1 / i$, there exists an integer $j$ greater than $m$ such that any subchain of $D_{j}$ contained in $D_{2}(u, v)$ and with end links in $f_{1}, f_{3}$ respectively has at least $n$ links.

We classify the links of $D_{j}$ that are contained in $D_{2}(u, v)$ as follows. Let $A_{1}$ be the set of those links of $D_{j}$ in $D_{2}(u, v)$ that are contained in $f_{1}$ or intersect $d(2)_{u}$. Let $A_{2}$ be the set of those links of $D_{j}$ in $D_{2}(u, v)$ that are contained in $f_{1}$ or intersect $d(2)_{v}$. Let $B_{i}(i=1,2)$ be the set of links of $D_{j}$ in $D_{2}(u, v)$ that are interior links of subchains of $D_{j}$ that have their end links in $A_{i}$ but have no interior link in $A_{1}+A_{2}$. Let $C$ be the set of links of $D_{j}$ in $D_{2}(u, v)$ that remain. Notice that each link in $C$ is an interior link of a subchain of $D_{j}$ that has one end link in $A_{1}$ and another in $A_{2}$ while no interior link of this subchain is an element of $A_{1}$ or $A_{2}$.

We define the chain $E$ as follows. The first link of $E$ is $A_{1}^{*}$; the last link is $A_{2}^{*}$; the second link contains $B_{1}^{*}$; the next to last link contains $B_{2}^{*}$; the links in $C$ are consolidated so that $E$ has the correct number of links.

This completes the proof of the theorem.

4. Description and some properties of the pseudo-arc $M$. Let $X$ and $Y$ be two points in a compact metric space. Let $W_{1}, W_{2}, \cdots$ be a sequence of chains from $X$ to $Y$ such that for each positive integer 1, (1) $W_{i+1}$ is crooked in $W_{i},(2)$ the mesh of $W_{i}$ is less than $1 / i$, and (3) any link of $W_{i}$ that contains 
a link of $W_{i+1}$ also contains the closure of that link. Then the intersection of $W_{1}^{*}, W_{2}^{*}, \cdots$ is the pseudo-arc $M$.

Throughout the rest of this paper, space is $M$ and therefore open sets are sets that are open in $M$. We shall also suppose that we have given a definite sequence $W_{1}, W_{2}, \cdots$ of chains satisfying conditions 1-3 whose intersection is $M$.

Some properties of the pseudo-arc $M$ that we shall make use of are contained in Theorems 2 and 3. Theorem 2 is due to Moise [6] and Theorem 3 to Bing [1].

THEOREM 2. The pseudo-arc is homeomorphic to each of its nondegenerate subcontinua.

Theorem 3. If the pseudo-arc $M$ is irreducible between the points $P$ and $Q$ there exists a sequence of chains $D_{1}, D_{2}, \ldots$ from $P$ to $Q$ such that for each positive integer $i, D_{i}$ is a consolidation of some $W_{m}, D_{i+1}$ is crooked in $D_{i}$, the mesh of $D_{i}$ is less than $1 / i$, and any link of $D_{i}$ that contains a link of $D_{i+1}$ also contains the closure of that link. In addition, if $D_{i}, D_{i^{\prime}}$, are consolidations of $W_{m}, W_{m^{\prime}}$, respectively, then $m$ is less than $m^{\prime}$ if $i$ is less than $i^{\prime}$.

Corollary. Suppose that the pseudo-arc $M$ is irreducible between the points $P$ and $Q$ and that $D$ is a chain from $P$ to $Q$ that covers $M$ and is a consolidation of some $W_{m}$. Then there exists a sequence of chains $D=E_{1}, E_{2}, \cdots$ from $P$ to $Q$ such that for each positive integer $i, E_{i}$ is a consolidation of some $W_{m}, E_{i+1}$ is crooked in $E_{i}$, the mesh of $E_{i+1}$ is less than $1 / i+1$, and any link of $E_{i}$ that contains a link of $E_{i+1}$ also contains the closure of that link. Also if $E_{i}, E_{i^{\prime}}$, are consolidations of $W_{m}, W_{m^{\prime}}$, respectively, then $m$ is less than $m^{\prime}$ if $i$ is less than $i^{\prime}$.

Proof. Suppose $D$ is a consolidation of $W_{k}$. Let $\epsilon$ be the Lebesgue number of $W_{k+1}$. Then there exists a sequence of chains $D_{1}, D_{2}, \cdots$ from $P$ to $Q$ satisfying the conclusions of Theorem 3 . Let $i$ be an integer such that $1 / i$ is less than minimum $(1, \epsilon)$. Then $D_{i}$ is contained in $W_{k+1}$ and by Lemma 2 , $D_{i}$ is crooked in $D$. Let $D_{i}=E_{2}$ and $D_{i+j}=E_{2+j}(j=1,2, \cdots)$. Then $E_{1}, E_{2}, \cdots$ is the desired sequence. (The fact that any link of $E_{1}$ that contains a link of $E_{i+1}$ also contains the closure of that link follows from the fact that each $E_{n}$ is a consolidation of some $W_{m}$.)

5. Some special chains. In this section we shall show how to construct some chains that cover the pseudo-arc $M$ in certain prescribed ways.

Lemma 3. Suppose $H_{1}, H_{2}, \cdots, H_{n}$ are nondegenerate disjoint subcontinua of the pseudo-arc $M$ such that $M$ is irreducible between each pair of them. Suppose further that $H_{i}$ is irreducible between the points $P_{i}$ and $Q_{i}(i=1,2, \cdots, n)$. Then for each positive number $\epsilon$, there exists an $\epsilon$-chain $D$ from $P_{1}$ to $Q_{n}$ that covers $M$ and such that $D$ is a consolidation of some $W_{m}, D\left(P_{i}, Q_{i}\right)$ contains $H_{i}(i=1,2, \cdots, n), D^{*}\left(P_{i}, Q_{i}\right)$ does not intersect $D^{*}\left(P_{j}, Q_{j}\right)$ if $i \neq j$ and $d_{Q_{1}}<d_{P_{2}}<d_{Q_{2}}<d_{P_{3}}<\cdots<d_{P_{n}}$. 
Proof. By Theorem 3, there exists a sequence of chains $D_{1}, D_{2}, \cdots$ from $P_{1}$ to $Q_{n}$ each of which covers $M$ and such that for each positive integer $i$, $D_{i}$ is a consolidation of some $W_{m}, D_{i+1}$ is crooked in $D_{i}$, the mesh of $D_{i}$ is less than $1 / i$ and any link of $D_{i}$ that contains a link of $D_{i+1}$ also contains the closure of that link.

Now the proof will proceed by an induction on $n$. Consider the case $n=2$. Since $H_{1}, H_{2}$ are disjoint pseudo-arcs (Theorem 2), there exist (by Theorem 3) $\epsilon$-chains $E_{i}=\left[e(i)_{1}, e(i)_{2}, \cdots, e(i)_{m_{1}}\right](i=1,2)$ from $P_{i}$ to $Q_{i}$ that cover $H_{i}$ and such that $E_{1}^{*}$ is disjoint from $E_{2}^{*}$.

Let $F=\left[f_{1}, f_{2}, \cdots, f_{u}\right]$ be $D_{j-1}$ where $j$ is chosen so large that the mesh of $F$ is less than the minimum of the Lebesgue numbers of $E_{1}$ and $E_{2}$ and any 4-linked subchain of $F$ one of whose links intersects $H_{i}$ is contained in a link of $E_{i}$.

Let $f_{r_{1}}$ be the last link of $F$ to contain $Q_{1}, f_{s_{1}}$ the last link of $F$ to intersect $H_{1}$, and $f_{t_{1}}$ the last link of $F$ such that $F\left(1, t_{1}\right)$ is contained in $E_{1}$. Then $5 \leqq r_{1} \leqq s_{1} \leqq t_{1}-3$. Similarly, let $f_{t_{2}}$ be the first link of $F$ such that $F\left(t_{2}, u\right)$ is contained in $E_{2}, f_{s_{2}}$ the first link of $F$ to intersect $H_{2}$, and $f_{r_{2}}$ the first link of $F$ to contain $P_{2}$. Then $t_{1}+1<t_{2} \leqq s_{2}-3<s_{2} \leqq r_{2} \leqq u-4$.

Now let $G=\left[g_{1}, g_{2}, \cdots, g_{v}\right]$ be $D_{j}$. Note that $G\left(=D_{j}\right)$ is crooked in $F\left(=D_{j-1}\right)$.

Let $g_{w_{1}}$ be the first link of $G$ to intersect $f_{t_{1}}, g_{w_{2}}$ the last link to intersect $f_{t_{2}}$. Since $G$ is crooked in $F, G\left(1, w_{1}\right)$ is the sum of three subchains $G\left(1, x_{1}\right)$, $G\left(x_{1}, y_{1}\right), G\left(y_{1}, w_{1}\right)$ such that $y_{1}$ is greater than $x_{1}, g_{x_{1}}$ is contained in $f_{t_{1-1}}$ and $g_{y_{1}}$ is contained in $f_{2}$. Similarly, $G\left(w_{2}, v\right)$ is the sum of subchains $G\left(w_{2}, y_{2}\right)$, $G\left(y_{2}, x_{2}\right), G\left(x_{2}, v\right)$ where $x_{2}$ is greater than $y_{2}, g_{y_{2}}$ is contained in $f_{u-1}$, and $g_{x_{2}}$ is contained in $f_{t_{2}+1}$. Since $t_{1} \geqq s+3, f_{t_{1}-1}$ follows $f_{s_{1}+1}$. And since $f_{s_{1}}$ was the last link of $F$ to intersect $H_{1}, g_{x_{1}}$ (which lies in $f_{t_{1-1}}$ ) does not intersect $H_{1}$. Hence, $G\left(1, x_{1}\right)$ contains $H_{1}$. Similarly, $G\left(x_{2}, v\right)$ contains $H_{2}$.

Now $t_{1}-1>r_{1}>2$ so $f_{r_{1}}$ lies between $f_{t_{1}-1}$ and $f_{2}$. And since $g_{x_{1}}, g_{y_{1}}$ are contained in $f_{t_{1}-1}, f_{2}$ respectively, there exists a link $g_{z_{1}}$ between $g_{x_{1}}$ and $g_{y_{1}}$ that is contained in $f_{r_{1}}$ and hence in $e(1)_{m_{1}}$. Similarly, there exists a link $g_{z_{2}}$ between $g_{y_{2}}$ and $g_{x_{2}}$ such that $g_{z_{2}}$ is contained in $e(2)_{1}$.

We now obtain the desired chain $D=\left[d_{1}, d_{2}, \cdots, d_{m}\right]$ as follows. For $i=1,2, \cdots, m_{1}, d_{i}$ is the sum of the links of $G\left(1, z_{1}\right)$ that are contained in the $i$ th link of $E_{1}$. The last $m_{2}$ links of $D$ are each the sum of the links of $G\left(z_{2}, v\right)$ that are contained in a link of $E_{2}$. The remaining (interior) links of $D$ are the links of $G\left(z_{1}+1, z_{2}-1\right)$. This proves the lemma for $n=2$.

Now suppose the lemma is true for all integers less than $n$. Then there exists an $\epsilon$-chain $E=\left[e_{1}, e_{2}, \cdots, e_{m}\right]$ from $P_{2}$ to $Q_{n}$ that covers $M$ and such that $E\left(P_{i}, Q_{i}\right)$ covers $H_{i}(i=2,3, \cdots, n), E^{*}\left(P_{i}, Q_{i}\right)$ does not intersect $E^{*}\left(P_{j}, Q_{j}\right)(i \neq j ; 2 \leqq i, j \leqq n)$ and $e_{Q_{2}}<e_{P_{3}}<e_{Q_{3}}<\cdots<e_{P_{n}}$.

Since the composants of $M$ are dense, there exists a point $X$ of $M-H_{1}$ that lies in the same composant as $H_{1}$ and only in the first link of $E$. Using 
the induction hypothesis (for the case $n=2$ ) we find a sequence of chains $F_{1}, F_{2}, \cdots$ from $P_{1}$ to $Q_{n}$ each of which covers $M$ and such that for each positive integer $k, F_{k}$ is a consolidation of some $W_{m}, F_{k}\left(P_{1}, Q_{1}\right)$ contains $H_{1}$, and the mesh of $F_{k}$ is less than $1 / k$. Then for some $k, F_{k}$ is contained in $E$, any link of $F_{k}$ containing $X$ does not intersect $H_{1}$, and $F_{k}\left(P_{1}, X\right)$ does not contain a point of $H_{2}+H_{3}+\cdots+H_{n}$. (The truth of this latter statement follows from the fact that $M$ is irreducible from $H_{1}$ to each of $H_{2}, H_{3}, \cdots, H_{n}$.)

Then if $f(k)_{x}$ is the first link of $F_{k}$ to contain $X$ the required chain $D$ is obtained as follows. The first $x-1$ links of $D$ are the links of $F_{k}(1, x-1)$ while the $x+i$ th link of $D(i=0,1, \cdots, m-1)$ is the sum of the links of $F_{k}\left(X, Q_{n}\right)$ that are contained in the $i+1$ st link of $E$. This completes the proof of the lemma.

Theorem 4 below shows that given a finite set of subcontinua of $M$ such that $M$ is irreducible between each pair, there exists an $\epsilon$-chain $D$ satisfying the conclusions of Lemma 3 and in addition contains another chain which follows a certain pattern in $D$ relative to these continua. The description of this pattern is contained in the definitions which follow.

If $D=\left[d_{1}, d_{2}, \cdots, d_{n}\right]$ is a chain from $P$ to $Q, R$ a point that lies only in $d_{i}(1<i<n)$, and $E=\left[e_{1}, e_{2}, \cdots, e_{m}\right]$ a chain from $P$ to $R$ that is contained in $D$, we say that $E$ follows a $v$-pattern to $R$ in $D$ if (1) for each link $e_{x}$ contained in $d_{i-j}$ there exists a link $e_{y}(y>x)$ contained in $d_{i+j}(j=1,2, \cdots$, minimum $(i-1, n-i)$ ), and (2) for each link $e_{x}$ contained in $d_{i+j+1}$ there exists a link $e_{y}(y>x)$ contained in $d_{i-j}(j=1,2, \cdots$, minimum $(i-1, n-i-1))$. (See Figure 1.)

Suppose $H$ is a proper subcontinuum of $M$ irreducible from $R$ to $S$ and $D=\left[d_{1}, d_{2}, \cdots, d_{n}\right]$ a chain covering $M$ such that $D(R, S)$ contains $H$ and $d_{1}<d_{R}<d_{n}$. Suppose $E=\left[e_{1}, e_{2}, \cdots, e_{m}\right]$ is a chain that is contained in $D$ whose end links are each subsets of only $d_{1}$, and such that $E(R, S)$ contains $H$. Let $e_{i}$ be the link of $E$ containing $R$. Then $E$ is said to follow a $v$-pattern in $D$ relative to $H, R, S$ provided $E$ is the sum of three subchains $E(1, i), E(i, j)$, $E(j, m)$ such that (1) $E(1, i)$ follows a $v$-pattern to $R$ in $D,(2) E(i, j)$ covers $H$, is contained in $D(R, S)$ and $e_{i}, e_{j}$ are each subsets of only $d_{R}$, and (3) $E(m, j)$ follows a $v$-pattern to $X$ in $D$ where $X$ is any point that is contained only in $e_{j}$.

Suppose $H_{1}, H_{2}, \cdots, H_{n}$ are nondegenerate disjoint subcontinua of the pseudo-arc $M$ and $H_{i}$ is irreducible between the points $P_{i}$ and $Q_{i}$ $(i=1,2, \cdots, n)$. Suppose $D$ is a chain from $P_{1}$ to $Q_{n}$ that covers $M$ and such that (1) $D\left(P_{i}, Q_{i}\right)$ contains $H_{i}(i=1,2, \cdots, n)$, (2) $D^{*}\left(P_{i}, Q_{i}\right)$ does not intersect $D^{*}\left(P_{j}, Q_{j}\right)$ if $i \neq j$, and (3) $d_{Q_{1}}<d_{P_{2}}<d_{Q_{2}}<\cdots<d_{P_{n}}$. Suppose $E=\left[e_{1}, e_{2}, \cdots, e_{m}\right]$ is a chain from $P_{1}$ to $Q_{n}$ that covers $M$, is contained in $D$, and such that $E\left(P_{i}, Q_{i}\right)$ contains $H_{i}(i=1,2, \cdots, n)$ with $e_{Q_{1}}<e_{P_{2}}<e_{Q_{2}}$ $<\cdots<e_{P_{n}}$. If $E$ is the sum of $n-1$ subchains $E\left(i_{1}, i_{2}\right), E\left(i_{2}, i_{3}\right), \cdots$, $E\left(i_{n-2}, i_{n-1}\right), E\left(i_{n-1}, m\right)$ where $e_{1}=e_{i_{1}}, e_{i_{2}}, \cdots, e_{i_{n-2}}$ are each contained in $d_{1}$ 
and $E\left(i_{j}, i_{j+1}\right)$ follows a $v$-pattern in $D$ relative to

$$
H_{j+1}, P_{j+1}, Q_{j+1}, \quad(j=1,2 \cdots, n-2)
$$

then $D$ is said to have property $S$ relative to $H_{1}, P_{1}, Q_{1}, \cdots, H_{n}, P_{n}, Q_{n}$ and $E$ is said to be a $V$-chain of $D$ relative to $H_{1}, P_{1}, Q_{1}, \cdots, H_{n}, P_{n}, Q_{n}$.

The next theorem shows that we can construct such chains $D$ and $E$ of arbitrarily small mesh provided only that $M$ is irreducible between each pair of $H_{1}, H_{2}, \cdots, H_{n}$.

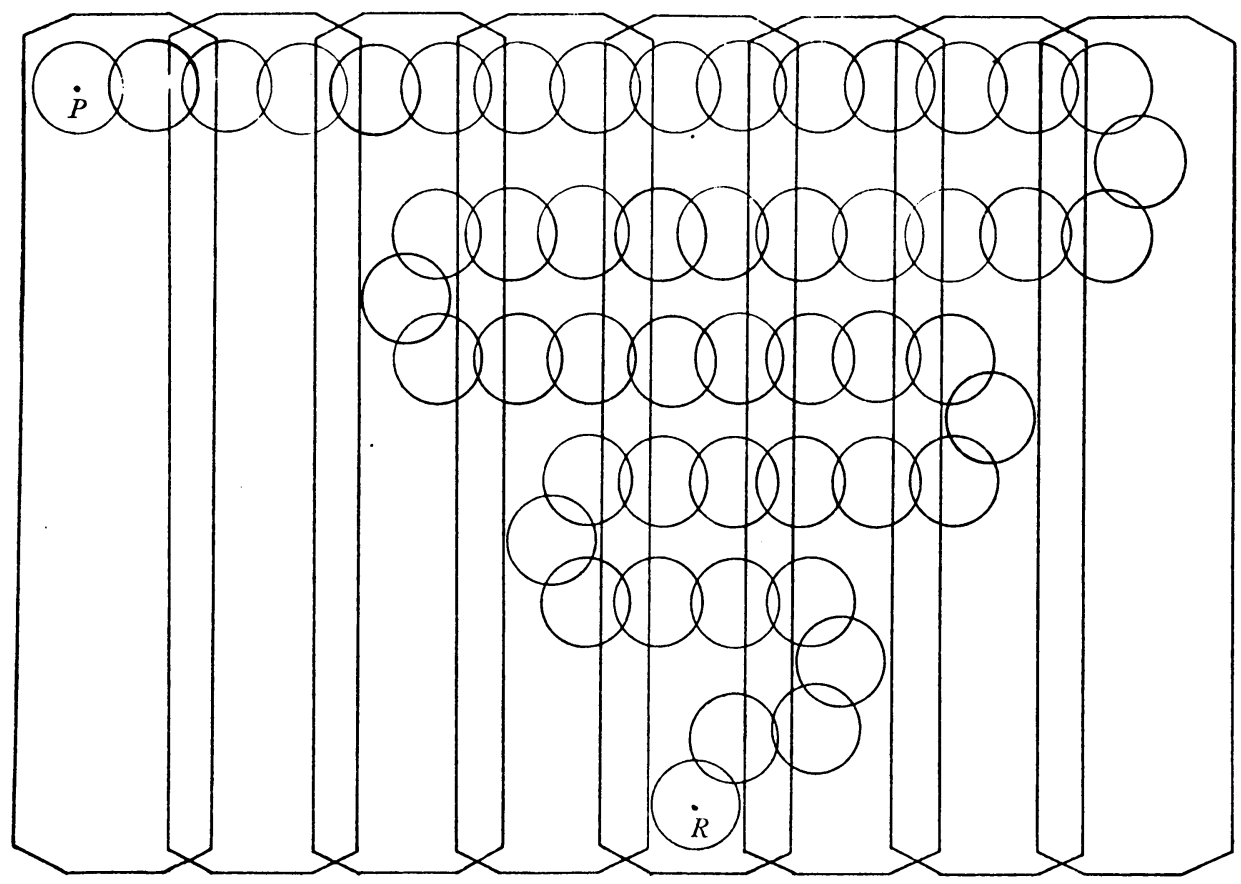

FIG. 1

Theorem 4. Suppose $H_{1}, H_{2}, \cdots, H_{n}(n \geqq 3)$ are nondegenerate proper subcontinua of the pseudo-arc $M$ such that $M$ is irreducible between each pair of them and such that $H_{\alpha}$ is irreducible between the points $P_{\alpha}, Q_{\alpha}(\alpha=1,2, \cdots, n)$. Then for each positive number $\epsilon$ there exist chains $D, G$ each of which is a consolidation of some $W_{m}$ and such that (1) $D$ covers $M$ and has mesh less than $\epsilon$, (2) $D$ has property $S$ relative to $H_{1}, P_{1}, Q_{1}, \cdots, H_{n}, P_{n}, Q_{n},(3) G$ covers $M$ and follows a $V$-pattern in $D$ relative to $H_{1}, P_{1}, Q_{1}, \cdots, H_{n}, P_{n}, Q_{n}$ and (4) any link of $D$ that contains a link of $G$ contains the closure of that link.

Proof. The proof will be by an induction on $n$. In proving the theorem for $n=3$, we shall label the steps and then perform analogous operations for the case of arbitrary $n$. 
Step 1. By Lemma 3, there exists a chain $C=\left[c_{1}, c_{2}, \cdots, c_{r}\right]$ from $P_{2}$ to $Q_{3}$ that is a consolidation of $W_{m_{1}}$, covers $M$, and such that $(1) \mu(C)<\epsilon$, (2) $C\left(P_{2}, Q_{2}\right)$ and $C\left(P_{3}, Q_{3}\right)$ contain $H_{2}$ and $H_{3}$ respectively, and (3) $C^{*}\left(P_{2}, Q_{2}\right)$ and $C^{*}\left(P_{3}, Q_{3}\right)$ are disjoint. Let $c_{8}$ be the link of $C$ that contains $Q_{2}$.

Step 2. By the corollary to Theorem 3 and Theorem 1 , there exists a chain $E^{\prime}$ from $P_{2}$ to $Q_{3}$ that is a consolidation of $W_{m_{2}}$, covers $M$ and follows the pattern $(1,1),(2,2),(3,1), \cdots,(m, x(m)), \cdots,(y(r), r)$ in $C$ where $y(r)$ is the number of links in $E^{\prime}, y(2)=4, y(r)=y(r-1)+4 r-5$ and $x(m)$ is the minimum value of $\{|m-z(n)|+1, n=1,2, \cdots\}$ where $z(1)=1$ and $z(n)=z(n-1)+2[n / 2]([n / 2]$ denotes the greatest integer in $n / 2)$. If $r=5$, $E^{\prime}$ follows the pattern indicated in Figure 2.

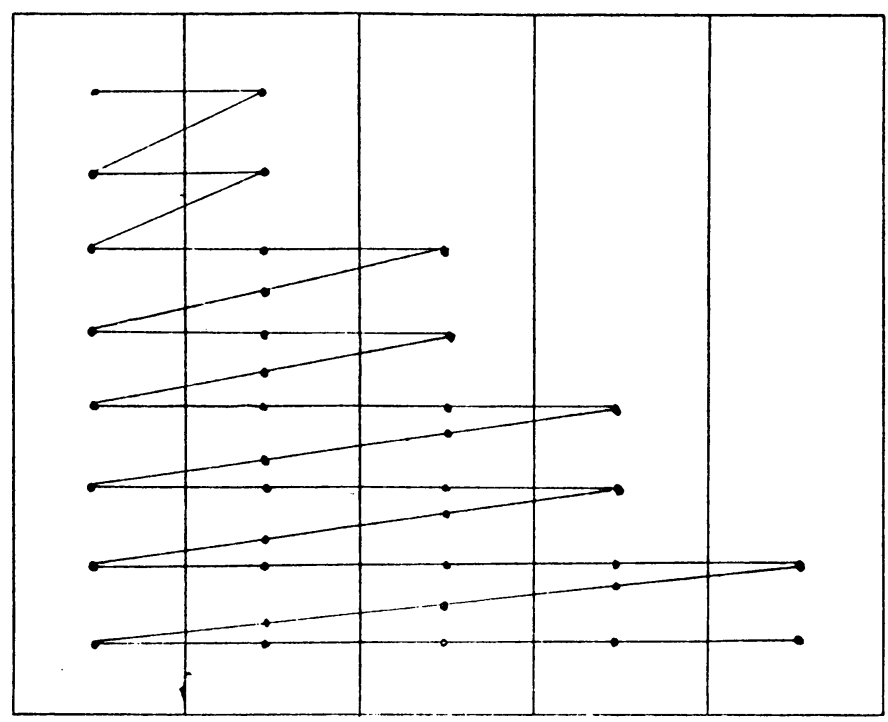

FIG. 2(2)

Note that any link of $C$ that contains a link of $E^{\prime}$ also contains the closure of that link. Also note that $E^{\prime}$ is the sum of $4 r-5$ subchains $E_{1}^{\prime}$, $E_{2}^{\prime}, \cdots, E_{4 r-5}^{\prime}$ where (1) $E_{4 k-3}^{\prime}, E_{4 k-2}^{\prime}, E_{4 k-1}^{\prime}, E_{4 k}^{\prime}(k=1,2, \cdots, r-2)$ each have $k+1$ links and follow the pattern $(1,1),(2,2), \cdots,(k+1, k+1)$ in $C$, (2) $E_{4 r-7}^{\prime}, E_{4 r-6}^{\prime}, E_{4 r-5}^{\prime}$ each have $r$ links and follow the pattern $(1,1),(2,2)$, $\cdots,(r, r)$ in $C$, and (3) the last link of $E_{j}^{\prime}$ is also the last link of $E_{j+1}^{\prime}$ and the first link of $E_{j+1}^{\prime}$ is also the first link of $E_{j+2}^{\prime}(j=1,3,5, \cdots, 4 r-7)$.

Step 3. By Lemma 3, there exists a chain $E^{\prime \prime}$ from $Q_{2}$ to $Q_{3}$ that is a consolidation of $W_{m_{\imath}}$, covers $M$, is contained in $E^{\prime}$ and such that $E^{\prime \prime}\left(Q_{2}, P_{2}\right)$ is contained in $C(1, s)$ and contains $H_{2}$. In addition, any link of $E^{\prime}$ that contains a link of $E^{\prime \prime}$ also contains the closure of that link.

(2) In this figure the five rectangles with disjoint interiors correspond to the links of $C$ while the broken line corresponds to the 1-nerve of $E^{\prime}$. 
Step 4. We now consolidate $E^{\prime \prime}$ so as to obtain a chain $E$ by the following scheme. If $e^{\prime \prime}$ is a link of $E^{\prime \prime}\left(Q_{2}, P_{2}\right)$ and $e^{\prime \prime}$ lies in the $j$ th link of $C(j=1,2, \cdots, s)$, let $e^{\prime \prime}$ be contained in the $s-(j-1)$ st link of $E$. If $e^{\prime \prime}$ is a link of $E^{\prime \prime}\left(P_{2}, Q_{3}\right)$ and $e^{\prime \prime}$ lies in the $j$ th link of $E^{\prime}$, let $e^{\prime \prime}$ be contained in the $s+(j-1)$ st link of $E$. Then $E=\left[e_{1}, e_{2}, \cdots, e_{t}\right]$ is a chain from $Q_{2}$ to $Q_{3}$ that is a consolidation of $W_{m_{3}}$, covers $M$, and is contained in $C$. Furthermore, contains $4 r-4$ subchains $E_{0}, E_{1}, \cdots, E_{4 r-5}$ where

(1) $E_{0}=E\left(P_{2}, Q_{2}\right)$ contains $H_{2}$ and follows the pattern $(1,1),(2,2), \cdots$, $(s, s)$ in $C$,

(2) the last link of $E_{j}$ is also the last link of $E_{j+1}$ and the first link of $E_{j+1}$ is also the first link of $E_{j+2}(j=1,3,5, \cdots, 4 r-7)$,

(3) the first link of $E_{0}$ is also the first link of $E_{1}$,

(4) $E_{4 k-3}, E_{4 k-2}, E_{4 k-1}, E_{4 k}(k=1,2, \cdots, r-2)$ each follow the pattern $(1,1),(2,2), \cdots,(k+1, k+1)$ in $C$, and

(5) $E_{4 r-7}, E_{4 r-6}, E_{4 r-5}$, each follow the pattern $(1,1),(2,2), \cdots,(r, r)$ in $C$. Finally, any link of $C$ that contains a link of $E$ also contains the closure of that link.

Step 5. Let $X_{1}$ and $X_{2}$ be two points of $M$ that are contained only in the last link of $E$ and $X_{3}$ a point of $M$ that lies only in the first link of $C$ such that $M$ is irreducible between each pair of $X_{1}, X_{2}, X_{3}, H_{1}, H_{2}, H_{3}$. Then by Lemma 3 , there exists a chain $F$ from $P_{1}$ to $Q_{3}$ such that (1) $F$ is contained in $E$ and covers $M$, (2) $F$ is a consolidation of $W_{m_{4}}$, (3) $F\left(P_{\alpha}, Q_{\alpha}\right)$ contains $H_{\alpha}(\alpha=1,2,3)$, (4) $f_{Q_{1}}<f_{X_{1}}<f_{P_{2}}<f_{Q_{2}}<f_{X_{2}}<f_{X_{3}}<f_{P_{3}}$, and (5) if any link of $E$ contains a link of $F$, it contains the closure of that link.

Step 6. We consolidate $F$ so as to obtain the chain $D$ as follows. If $f_{X_{1}}$ is the $(i+1)$ st link of $F$, let the $j$ th link of $D$ be the $j$ th link of $F(j=1,2, \cdots, i)$. If $f$ is a link of $F\left(X_{1}, X_{2}\right)$ and $f$ lies in the $j$ th link of $E_{k}(k=0,1, \cdots, 4 r-5)$, $f$ is a subset of the $i+r \pm(j-1)$ st link of $D$ where the plus sign is used if $k=0$ or if $k \equiv 1$ or $2 \bmod 4$ and the minus sign is used otherwise. If $f$ is a link of $F\left(X_{2}, X_{3}\right)$ and $f$ is a subset of the $j$ th link of $C, f$ is contained in $d_{i+r-(j-1)}$. If $f$ is a link of $F\left(X_{3}, Q_{3}\right)$ and $f$ lies in the $j$ th link of $C, f$ is contained in $d_{i+r+(j-1)}$.

Then $D=\left[d_{1}, d_{2}, \cdots, d_{i+2 r-1}\right]$ is an $\epsilon$-chain from $P_{1}$ to $Q_{3}$ that is a consolidation of $W_{m_{4}}$, covers $M$, and such that $D\left(P_{\alpha}, Q_{\alpha}\right)$ contains $H_{\alpha}(\alpha=1,2,3)$, $D^{*}\left(P_{\alpha}, Q_{\alpha}\right)$ does not intersect $D^{*}\left(P_{\beta}, Q_{\beta}\right)$ if $\alpha \neq \beta$ and $d_{P \alpha}<d_{Q \alpha}(\alpha=1,2,3)$. In the steps that follow, we shall show that $D$ has property $S$ relative to $H_{1}, P_{1}, Q_{1}, \cdots, H_{3}, P_{3}, Q_{3}$.

Step 7. Here we consolidate subchains of $F$ and obtain chains $G_{1}^{\prime}, G_{2}^{\prime}$, $G_{3}^{\prime}, G_{4}^{\prime}$. These are then added together (end-to-end) to obtain a new chain $G^{\prime}$. We then show (Steps 8 and 9) that $G^{\prime}$ is close to being a $V$-chain of $D$ relative to $H_{1}, P_{1}, Q_{1}, \cdots, H_{3}, P_{3}, Q_{3}$. Then using $G^{\prime}$ we construct a chain $G$ (Step 10) and show (Step 11) that it is a $V$-chain of $D$ relative to $H_{1}, P_{1}$, $Q_{1}, \cdots, H_{3}, P_{3}, Q_{3}$. 
If $f_{j}$ is the $j$ th link of $F(1, i)(j=1,2, \cdots, i)$, let $G_{1}^{\prime}$ be the chain whose $j$ th link is $f_{j}$.

If $f$ is a link of $F\left(X_{1}, Q_{2}\right)$ and $f$ lies in the $j$ th link of $E=\left[e_{1}, e_{2}, \cdots, e_{t}\right]$, let $f$ be contained in the $t-j+1$ st link of $G_{2}^{\prime}$.

If $f$ is a link of $F\left(Q_{2}, X_{2}\right)$ and $f$ lies in the $j$ th link of $E$, let $f$ be contained in the $j$ th link of $G_{3}^{\prime}$.

If $f$ is a link of $F\left(X_{2}, Q_{3}\right)$ and $f$ lies in the $j$ th link of $D(j=i+1, i+2, \cdots$, $i+2 r-1), f$ is a subset of the $(j-i)$ th link of $G_{4}^{\prime}$.

Let $G^{\prime}$ be the chain whose first $i$ links are the links of $G_{1}^{\prime}$, whose $i+1$ st link is the first link of $G_{2}^{\prime}$, whose last link is the last link of $G_{4}^{\prime}$ and whose remaining links are interior links of $G_{2}^{\prime}, G_{3}^{\prime}, G_{4}^{\prime}$ or the sum of the last link of $G_{\alpha}^{\prime}$ and the first link of $G_{\alpha+1}^{\prime}(\alpha=2,3)$.

Then $G^{\prime}=\left[g_{1}^{\prime}, g_{2}^{\prime}, \cdots, g_{u}^{\prime}\right]$ is a chain from $P_{1}$ to $Q_{3}$ that is a consolidation of $W_{m_{4}}$, covers $M$, is contained in $D$ and such that $G^{\prime}\left(P_{\alpha}, Q_{\alpha}\right)$ contains $H_{\alpha}(\alpha=1,2,3), G^{*}\left(P_{\alpha}, Q_{\alpha}\right)$ does not intersect $G^{*}\left(P_{\beta}, Q_{\beta}\right)$ if $\alpha \neq \beta$, and $g_{P_{\alpha}}^{\prime}<g_{Q_{\alpha}}^{\prime}(\alpha=1,2,3)$.

Step 8. Before seeing how $G^{\prime}$ comes close to following a $V$-pattern in $D$, we shall show that $G^{\prime}$ follows a unique pattern in $D$. This will follow immediately after we show that the patterns follows by $G_{1}^{\prime}, G_{2}^{\prime}, G_{z}^{\prime}$ and $G_{4}^{\prime}$ are each unique.

The pattern followed by $G_{1}^{\prime}$ is unique since the links of $G_{1}^{\prime}$ are also links of $D$.

Let $g^{\prime}$ be any link of $G_{2}^{\prime} \cdot g^{\prime}$ consists of all the links of $F\left(X_{1}, Q_{2}\right)$ that are contained in some particular link, call it $e$, of $E$. Since $X_{1}, Q_{2}$ are contained only in the last and first links respectively of $E$, so are $f_{X_{1}}, f_{Q_{2}}$ contained only in these links. Hence, each link of $E$ (in particular say $e$ ) contains some link of $F\left(X_{1}, Q_{2}\right)$ that is contained in no other link of $E$. Let $f$ be a link of $F\left(X_{1}, Q_{z}\right)$ that is contained only in $e$. (Then $f$ is also a subset of $g^{\prime}$.) But by the definition of $D, f$ is then contained in only a single link of $D$. Hence since $g^{\prime}$ contains $f, g^{\prime}$ can be contained in only a single link of $D$.

A similar argument applies to $G_{3}^{\prime}$.

Now let $g^{\prime}$ be any link of $G_{4}^{\prime}$. It consists of all links of $F\left(X_{2}, Q_{3}\right)$ that are contained in some particular link $d$ of $D(i+1, i+2 r-1)$. But since $f_{X_{2}}, f_{Q_{2}}$ lie only in the first and last links respectively of $D(i+1, i+2 r-1), d$ must contain some link $f$ of $F\left(X_{2}, Q_{3}\right)$ that is contained in no other link of $D$. Then $g^{\prime}$ contains $f$ and hence can be contained in only one link of $D$.

Therefore $G^{\prime}$ follows a unique pattern in $D$.

Step 9. We now begin to show that $G^{\prime}$ comes close to being a $V$-chain of $D$ relative to $H_{1}, P_{1}, Q_{1}, \cdots, H_{3}, P_{3}, Q_{3}$. Exactly how close will become apparent as we describe the pattern that $G^{\prime}$ follows in $D$.

$G^{\prime}\left(P_{1}, P_{2}\right)$ follows a v-pattern to $P_{2}$ in $D$. To see this first suppose that $g_{m}^{\prime}$ is a link of $G^{\prime}\left(P_{1}, P_{2}\right)$ that lies in the $(i+r-j)$ th link of $D(j=1,2, \cdots, r-1)$. 
We must prove that there exists a link $g_{n}^{\prime}$ of $G^{\prime}\left(P_{1}, P_{2}\right)$ with $n>m$ and such that $g_{n}^{\prime}$ is contained in the $(i+r+j)$ th link of $D$.

Since $g_{m}^{\prime}$ is a link of $G^{\prime}\left(P_{1}, P_{2}\right)$ and $j \leqq r-1, g_{m}^{\prime}$ is a link of $G_{2}^{\prime}$ and hence is the sum of the links of $F\left(X_{1}, Q_{2}\right)$ that are contained in a particular link of $E$. By the definition of $D$, the links of $F\left(X_{1}, Q_{2}\right)$ that are subsets of the $(i+r-j)$ th link of $D(j=1,2, \cdots, r-1)$ are the links that are contained in the $(j+1)$ st links of those $E_{k}$ such that $k>0$ and $k \equiv 3$ or $4 \bmod 4$. Since $k>0$ and $K \equiv 3$ or $4 \bmod 4, E_{k-2}$ exists and (by the definition of $E_{1}, E_{2}, \cdots$, $\left.E_{4_{r-5}}\right)$ has the same number of links as $E_{k}$. Since the links of $E_{k-2}$ precede the links of $E_{k}$ in $E$, the $(j+1)$ st link of $E_{k-2}$ precedes the $(j+1)$ st link of $E_{k}$ in $E$. Then if $g_{n}^{\prime}$ is the link of $G_{2}^{\prime}$ that consists of the sum of the links of $F\left(X_{1}, Q_{2}\right)$ in the $(j+1)$ st link of $E_{k-2}, g_{n}^{\prime}$ follows $g_{m}^{\prime}$ in $G_{2}^{\prime}$ by the definition of $G_{2}^{\prime}$. And since $k-2 \equiv 1$ or $2 \bmod 4$, the links of $F\left(X_{1}, Q_{2}\right)$ that lie in the $(j+1)$ st link of $E_{k-2}$ are contained in the $(i+r+j)$ th link of $D$. That is, $g_{n}^{\prime}$ is contained in $d_{i+r+j}$.

Now suppose $g_{m}^{\prime}$ is a link of $G^{\prime}\left(P_{1}, P_{2}\right)$ that lies in the $(i+r+j+1)$ st link of $D(j=1,2, \cdots, r-2)$. We must show that there exists a link $g_{n}^{\prime}$ of $G^{\prime}\left(P_{1}, P_{2}\right)$ with $n>m$, and such that $g_{n}^{\prime}$ is contained in $d_{i+r-j}$.

Since $g_{m}^{\prime}$ is a link of $G^{\prime}\left(P_{1}, P_{2}\right)$ and $j \geqq 1, g_{m}^{\prime}$ is a link of $G_{2}^{\prime}$ and hence the sum of the links of $F\left(X_{1}, Q_{2}\right)$ that are contained in some particular link of $E$. By the definition of $D$, the links of $F\left(X_{1}, Q_{2}\right)$ that are contained in the $(i+r+j+1)$ st link of $D$ are those that are subsets of the $(j+2)$ nd links of those $E_{k}$ for which $k=0$ or $k \equiv 1$ or $2 \bmod 4$. Hence, $g_{m}^{\prime}$ is the sum of the links of $F\left(X_{1}, Q_{2}\right)$ that are contained in the $(j+2)$ nd link of some particular $E_{k}$ where $k=0$ or $k \equiv 1$ or $2 \bmod 4$. But since $j \geqq 1, j+2 \geqq 3$ and thus $k$ is not 1 or 2 since $E_{1}$ and $E_{2}$ each have only two links. Also since $P_{2}$ is contained in the first link of $E_{0}, g_{m}^{\prime}$ (being a link of $G^{\prime}\left(P_{1}, P_{2}\right)$ ) is not the sum of the links of $F\left(X_{1}, Q_{2}\right)$ that lie in the $(j+2)$ nd link of $E_{0}$. Thus $k \geqq 5$ and $k-2$ exists and $k-2 \equiv 3$ or $4 \bmod 4$. Since $E_{k-2}$ has only one less link than $E_{k}, E_{k-2}$, has a $(j+1)$ st link and this link precedes the $(j+2)$ nd link of $E_{k}$ in $E$. Hence the sum of the links of $F\left(X_{1}, Q_{2}\right)$ that are contained in the $(j+1)$ st link of $E_{k-2}$ is a link, call it $g_{n}{ }^{\prime}$, of $G_{2}^{\prime}$ that follows $g_{m}^{\prime}$ in $G_{2}^{\prime}$. And by the definition of $D$, the links of $F\left(X_{1}, Q_{2}\right)$ that are contained in $g_{n}^{\prime}$ are subsets of the $(i+r-j)$ th link of $D$. Hence, $g_{n}^{\prime}$ is contained in $d_{i+r-j}$. This completes the proof that $G^{\prime}\left(P_{1}, P_{2}\right)$ follows a $v$-pattern to $P_{2}$ in $D$.

Next we note that since $G^{\prime}\left(P_{2}, Q_{2}\right)$ is a consolidation of the links of $F\left(X_{1}, Q_{2}\right)$ in $E_{0}, G^{\prime}\left(P_{2}, Q_{2}\right)$ is contained in $D\left(P_{2}, Q_{2}\right)$.

Since $f_{Q_{2}}, f_{X_{2}}$ lie in only the first and last links of $E$ respectively, there exists a point $X_{4}$ of $M$ such that $f_{X_{4}}$ is a link of $F\left(Q_{2}, X_{2}\right)$ and is contained in the first link of $E_{0}$. Then $G^{\prime}\left(Q_{2}, X_{4}\right)$ is contained in $D\left(P_{2}, Q_{2}\right)$ since it is a consolidation of the links of $F\left(Q_{2}, X_{2}\right)$ that lie in $E_{0}$. In addition, $g_{X_{4}}^{\prime}$ is contained in $d_{P_{2}}$.

We will finally consider the pattern followed by $G^{\prime}\left(X_{4}, Q_{3}\right)$ in $D$. Since 
$G^{\prime}\left(X_{4}, Q_{3}\right)$ is contained in $D(i+1, i+2 r-1), G^{\prime}\left(Q_{3}, X_{4}\right)$ does not follow a $v$-pattern to $X_{4}$ in $D$. However, by using arguments analogous to those used in proving that $G^{\prime}\left(P_{1}, P_{2}\right)$ follows a $v$-pattern to $P_{2}$ in $D$ we can prove the following: (1) if $g_{m}^{\prime}$ is a link of $G^{\prime}\left(X_{4}, Q_{3}\right)$ that is contained in the $(i+r-j)$ th link of $D(j=1,2, \cdots, r-1)$, then there exists a link $g_{n}^{\prime}$ of $G^{\prime}\left(X_{4}, Q_{3}\right)$ with $n<m$ and such that $g_{n}^{\prime}$ is a subset of the $(i+r+j)$ th link of $D$; and (2) if $g_{m}^{\prime}$ is a link of $G^{\prime}\left(X_{4}, Q_{3}\right)$ that is contained in the $(i+r+j+1)$ st link of $D(j=1,2, \cdots, r-2)$, then there exists a link $g_{n}^{\prime}$ of $G^{\prime}\left(X_{4}, Q_{3}\right)$ with $n<m$ and such that $g_{n}^{\prime}$ is a subset of the $(i+r-j)$ th link of $D$.

Figure 3 indicates roughly the pattern that $G^{\prime}$ follows in $D$ and the relative positions of $P_{\alpha}, Q_{\alpha}, X_{\alpha}(\alpha=1,2,3)$ and $X_{4}$.

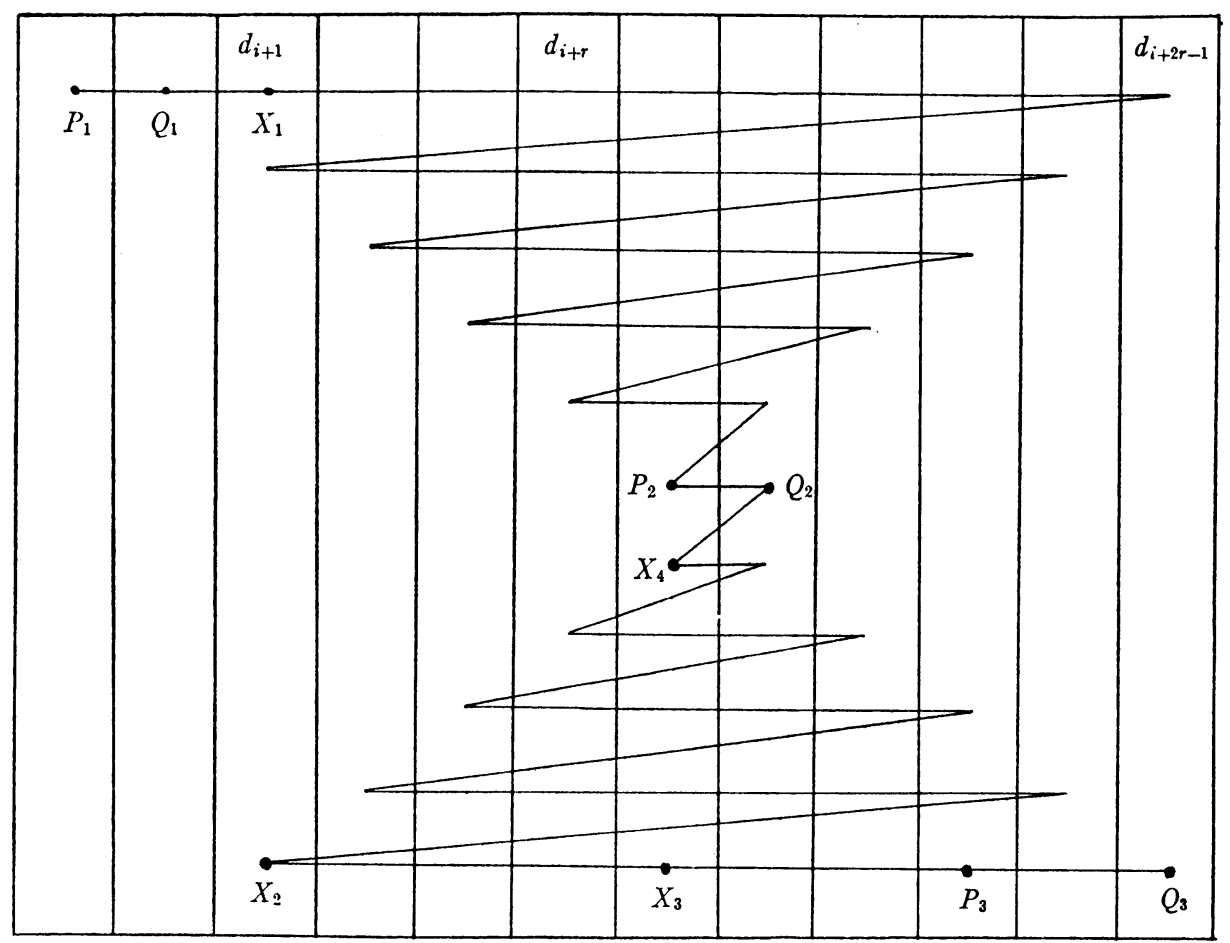

FIG. 3

Step 10. In this step we construct a chain $G$ from $P_{1}$ to $Q_{3}$ that follows a $V$-pattern in $D$ relative to $H_{1}, P_{1}, Q_{1}, \cdots, H_{3}, P_{3}, Q_{3}$.

Since $G^{\prime}$ follows a unique pattern in $D$ and the composants of $M$ are dense, each link $g_{j}^{\prime}$ of $G^{\prime}$ contains a point $Z_{j}$ that is contained only in the unique link of $D$ containing $g_{j}^{\prime}$ and such that $M$ is irreducible between each pair of the points $P_{1}, P_{2}, P_{3}, X_{1}, X_{2}, X_{3}, X_{4}, Z_{1}, Z_{2}, \cdots, Z_{u}$. In addition, there exist points $Y_{1}, Y_{2}$ contained in only the last link of $G^{\prime}$ and the first link of $D$ 
respectively such that $M$ is irreducible between each pair of the points $P_{1}, P_{2}, P_{3}, X_{1}, \cdots, X_{4}, Z_{1}, Z_{2}, \cdots, Z_{u}, Y_{1}, Y_{2}$.

Then by Lemma 3 there exists a chain $G^{\prime \prime}$ from $P_{1}$ to $Q_{3}$ such that (1) $G^{\prime \prime}$ is a consolidation of $W_{m_{b}}$, (2) $G^{\prime \prime}$ covers $M$ and is contained in $G^{\prime},(3) G^{\prime \prime}\left(P_{1}, Y_{1}\right)$ contains $H_{1}+H_{2}+X_{4}$, (4) $G^{\prime \prime}\left(Y_{2}, Q_{3}\right)$ contains $H_{3}$, and (5) $g_{Z_{i}}^{\prime \prime}$ is a link of $G^{\prime \prime}\left(P_{1}, Y_{1}\right)(j=1,2, \cdots, u)$. Note that if any link of $G^{\prime}$ contains a link of $G^{\prime \prime}$, then it also contains the closure of that link.

We now define chains $G_{1}, G_{2}, G_{3}$ and then add them together (end-to-end) to obtain the desired chain $G$.

Let $G_{1}$ be the chain whose $j$ th $\operatorname{link}(j=1,2, \cdots, u)$ is the sum of the links of $G^{\prime \prime}\left(P_{1}, Y_{1}\right)$ that are contained in the $j$ th link of $G^{\prime}$.

If $g^{\prime \prime}$ is a link of $G^{\prime \prime}\left(Y_{1}, Y_{2}\right)$ and $g^{\prime \prime}$ is in the $j$ th link of $D(j=1,2, \cdots$, $1+2 r-1), g^{\prime \prime}$ is a subset of the $(i+2 r-j)$ th link of $G_{2}$.

If $g^{\prime \prime}$ is a link of $G^{\prime \prime}\left(Y_{2}, Q_{3}\right)$ and $g^{\prime \prime}$ is in the $j$ th link of $F(j=1,2, \cdots$, $i+2 r-1), g^{\prime \prime}$ is a subset of the $j$ th link of $G_{3}$.

Then $G$ is the chain whose first and last links are the first and last links of $G_{1}$ and $G_{3}$ respectively and whose interior links are the interior links of $G_{\alpha}(\alpha=1,2,3)$ or the sum of the last link of $G_{\alpha}$ and the first link of $G_{\alpha+1}(\alpha=1,2)$. Note that $G$ is a consolidation of $W_{m_{b}}$ and that if any link of $D$ contains a link of $G$, it also contains the closure of that link.

Step 11. In the last step we show that $G$ follows a $V$-pattern in $D$ relative to $H_{1}, P_{1}, Q_{1}, \cdots, H_{3}, P_{3}, Q_{3}$.

Since the $j$ th link of $G^{\prime}(j=1,2, \cdots, u)$ contains $z_{Z j}^{\prime \prime}$, the $j$ th link of $G_{1}$ contains $g_{z_{j}}$ and hence the $j$ th link of $G_{1}$ is contained in only one link of $D$. Hence, $G_{1}$ follows a unique pattern in $D$ and this pattern is identical to that followed by $G^{\prime}$ in $D$. And since $G_{1}$ contains the links of $G^{\prime}\left(P_{1}, Y_{1}\right), G_{1}$ contains $H_{1}+H_{2}+X_{4}$.

Therefore $G\left(P_{1}, P_{2}\right)$ follows a $v$-pattern to $P_{2}$ in $D$ and $G\left(P_{2}, Q_{2}\right)$ and $G\left(Q_{2}, X_{4}\right)$ are contained in $D\left(P_{2}, Q_{2}\right)$. It remains to show that $G\left(Y_{2}, X_{4}\right)$ follows a v-pattern to $X_{4}$ in $D$.

Suppose $g_{m}$ is a link of $G\left(Y_{1}, X_{4}\right)$ that lies in the $(i+r-j)$ th link of $D(j=1,2, \cdots, r-1)$. (We shall consider the case where $g_{m}$ is a link of $G\left(Y_{2}, Y_{1}\right)$ later.) Then $g_{m}$ is a link of $G_{1}$ and hence is contained in a link $g_{m^{\prime}}^{\prime}$ of $G^{\prime}\left(X_{4}, Q_{3}\right)$. Since $G_{1}$ and $G^{\prime}$ follow identical patterns in $D, g_{m^{\prime}}^{\prime}$ is also contained in the $(i+r-j)$ th link of $D$. In Step 9, we proved that in this case there exists a link $g_{n}^{\prime}$ of $G^{\prime}\left(X_{4}, Q_{3}\right)$ with $n^{\prime}<m^{\prime}$ such that $g_{n^{\prime}}^{\prime}$ is a subset of the $(i+r+j)$ th link of $D$. Then the sum of the links of $G^{\prime \prime}\left(P_{1}, Y_{2}\right)$ that lie in $g_{n^{\prime}}^{\prime}$ will constitute a link $g_{n}$ of $G\left(Y_{1}, X_{4}\right)$ such that $g_{n}$ will be a subset of $(i+r+j)$ th link of $D$ and follow $g_{m}$ in the chain $G\left(Y_{1}, X_{4}\right)$.

In case $g_{m}$ is a link of $G\left(Y_{2}, Y_{1}\right)$ and is contained in the $(i+r-j)$ th link of $D(j=1,2, \cdots, r-1)$, there exists a link $g_{\bar{m}}$ of $G\left(Y_{1}, X_{4}\right)$ that follows $g_{m}$ in $G\left(Y_{1}, X_{4}\right)$ and is contained in the same link of $D$. Then a repetition of the above argument yields a link $g_{n}$ of $G\left(Y_{1}, X_{4}\right)$ (and therefore of $G\left(Y_{2}, X_{4}\right)$ ) that 
follows $g_{\bar{m}}$ (and therefore $\left.g_{m}\right)$ in $G\left(Y_{2}, X_{4}\right)$ and is contained in the $(i+r+j)$ th link of $D$.

In the same way we can show that if $g_{m}$ is a link of $G\left(Y_{2}, X_{4}\right)$ that is contained in the $(i+r+j+1)$ st link of $D(j=1,2, \cdots, r-2)$, then there exists a link $g_{n}$ of $G\left(Y_{2}, X_{4}\right)$ that follows $g_{m}$ in $G\left(Y_{2}, X_{4}\right)$ and such that $g_{n}$ is contained in the $(i+r-j)$ th link of $D$.

Hence, $G$ is a $V$-chain of $D$ relative to $H_{1}, P_{1}, Q_{1}, \cdots, H_{3}, P_{3}, Q_{3}$ and the proof of Theorem 4 for the case $n=3$ is completed.

Now suppose the theorem is true for all integers less than $n$. We shall indicate the procedure that will yield an $\epsilon$-chain $D$ that has property $S$ relative to $H_{1}, P_{1}, Q_{1}, \cdots, H_{n}, P_{n}, Q_{n}$.

Step $1^{\prime}$. By the induction hypothesis, there exists an $\epsilon$-chain $C$ from $P_{2}$ to $Q_{n}$ that covers $M$ and has property $S$ relative to $H_{2}, P_{2}, Q_{2}, \cdots, H_{n}, P_{n}, Q_{n}$.

Steps $2^{\prime}, 3^{\prime}, 4^{\prime}$. Just as before we construct a chain $E$ from $Q_{2}$ to $Q_{n}$ that covers $M$, is contained in $C$, and in addition contains $4 r-4$ subchains $E_{0}, E_{1}, \cdots, E_{4 r-5}$ satisfying conditions $1,2,3,4,5$ of Step 4.

Step $5^{\prime}$. Using the same procedure as in Step 5 we construct a chain $F$ from $P_{1}$, to $Q_{n}$ such that (1) $F$ is contained in $E$ and covers $M$, (2) $F\left(P_{\alpha}, Q_{\alpha}\right)$ contains $H_{\alpha}(\alpha=1,2, \cdots, n)$, (3) $f_{Q_{1}}<f_{X_{1}}<f_{P_{2}}<f_{Q_{2}}<f_{X_{2}}<f_{X_{3}}$, and (4) $F\left(X_{3}, Q_{n}\right)$ contains $H_{3}+H_{4}+\cdots+H_{n}$.

Step 6'. This step is almost identical to Step 6. The only change necessary is to note that the instructions on how to consolidate $F\left(X_{3}, Q_{3}\right)$ are now to be regarded as instructions on how to consolidate $F\left(X_{3}, Q_{n}\right)$.

This gives us the chain $D$ and essentially the same methods used in Steps 7-11 show that $D$ has property $S$ relative to $H_{1}, P_{1}, Q_{1}, \cdots, H_{n}, P_{n}, Q_{n}$.

6. Extending homeomorphisms. In this section we consider the problem of extending a homeomorphism that is defined between certain subsets of the pseudo-arc. In particular, Theorem 7 gives a necessary and sufficient condition that the homeomorphism can be extended to the entire pseudo-arc in case the subsets are closed and contain at most a finite number of components.

Theorem 5 below is stated without proof. It is a result of Bing's [1] we shall use to obtain the desired homeomorphisms.

Theorem 5. Suppose $M$ is the pseudo-arc; $\epsilon_{1}, \epsilon_{2}, \ldots$ is a sequence of positive numbers with a finite sum; and $D_{i, 1}, D_{i, 2}, \cdots(i=1,2)$ is a sequence of chains such that for each positive integer $j$, (1) $D_{i, j}$ covers $M$, (2) each link of $D_{i, j}$ intersects $M,(3) \mu\left(D_{i, j}\right)$ is less than $\epsilon_{j}$, (4) $D_{1, j}$ and $D_{2, j}$ each have the same number of links, and (5) if the mth link of $D_{i, j 1}$ intersects the nth link of $D_{i, j}$, then the distance between the mth link of $D_{i^{\prime}, j 1}$ and the nth link of $D_{i^{\prime}, j}$ is less than $\epsilon_{j}\left(i, i^{\prime}=1,2\right)$. Then these sequences of chains induce a homeomorphism $T$ of $M$ onto $M$ such that if $P$ is a point of $M$ that is contained in the mth link of $D_{i, j}$, then $T(P)$ is contained in the mth link of $D_{i^{\prime}, j}\left(i, i^{\prime}=1,2\right)$. 
Lemma 4. Suppose $H_{1,1}, H_{1,2}, \cdots, H_{1, n}(n \geqq 3)$ are nondegenerate, disjoint subcontinua of the pseudo-arc $M$ such that $M$ is irreducible between each pair of them and $H_{1, j}$ is irreducible between the points $P_{1, j}, Q_{1, j}(j=1,2, \cdots, n)$. Suppose also that $T$ is a homeomorphism of $H_{1,1}+H_{1,2}+\cdots+H_{1, n}$ onto $H_{2,1}$ $+H_{2,2}+\cdots+H_{2, n}$ such that $T\left(H_{1, j}\right)=H_{2, j}, \quad T\left(P_{1, j}\right)=P_{2, j}, \quad T\left(Q_{1, j}\right)$ $=Q_{2, j}(j=1,2, \cdots, n)$ where $H_{2,1}, H_{2,2}, \cdots, H_{2, n}$ are disjoint subcontinua of $M$ and $M$ is irreducible between each pair of them. Then for each positive number $\epsilon$, there exist chains $D_{1}, D_{2}$ such that (1) $D_{i}$ is a consolidation of some $W_{m_{i}}$, (2) $D_{i}$ is a chain from $P_{i, 1}$ to $Q_{i, n}$ that covers $M$, (3) $\mu\left(D_{2}\right)$ is less than $\epsilon$, (4) $D_{i}$ has property $S$ relative to $H_{i, 1}, P_{i, 1}, Q_{i, 1}, \cdots, H_{i, n}, P_{i, n}, Q_{i, n}(i=1,2),(5) D_{1}$ has the same number of links as $D_{2},(6) P_{1, j}\left(Q_{1, j}\right)$ and $P_{2, j}\left(Q_{2, j}\right)$ lie in corresponding links of $D_{1}, D_{2}$ respectively, (7) $D_{1}\left(P_{1, j}, Q_{1, j}\right)$ has the same number of links as $D_{2}\left(P_{2, j}, Q_{2, j}\right)(j=1,2, \cdots, n),(8) D_{1}\left(Q_{1, j}, P_{1, j+1}\right)$ has the same number of links as $D_{2}\left(Q_{2, j}, P_{2, j+1}\right)(j=1,2, \cdots, n)$, and (9) if the kth link of $D_{1}$ intersects $H_{1,1}+H_{1,2}+\cdots+H_{1, n}$, then $T$ maps their common part into the kth link of $D_{2}$.

Proof. The proof of this lemma is very similar to that of Theorem 4. In fact, the constructions described there will, with only minor modification, also yield the required chains $D_{1}$ and $D_{2}$. Rather than repeat the proof of Theorem 4 in its entirety therefore, we shall simply indicate what added precautions must be taken at each step. The notation used here will correspond to that used in the proof of Theorem 4 . Again we consider first the case $n=3$.

We construct the chain $D_{2}$ by the procedure described in Theorem 4 such that $\mu\left(D_{2}\right)$ is less than $\epsilon$.

Let $\epsilon^{\prime}$ be the Lebesgue number of $D_{2}$ and let $\delta$ be chosen so that if $X, Y$ are points of $H_{1,1}+H_{1,2}+H_{1,3}$ whose distance apart is less than $\delta$, then the distance from $T(X)$ to $T(Y)$ is less than $\epsilon^{\prime}$.

By using Lemma 3, we find a chain $C^{\prime}$ from $P_{1,2}$ to $Q_{1,3}$ that is a consolidation of some $W_{m}$, and covers $M$, and such that (1) $\mu\left(C^{\prime}\right)<\delta$, (2) $C^{\prime}\left(P_{1, j}, Q_{1, j}\right)$ covers $H_{1, j}(j=2,3)$, and $(3) C^{\prime}\left(Q_{1,2}, P_{1,3}\right)$ has at least as many links as $D_{2}\left(Q_{2,2}, P_{2,3}\right)$.

We now consolidate $C^{\prime}$ to obtain a chain $C$ which will play the role of the chain $C$ obtained in Step 1 of the proof of Theorem 4. Suppose $D_{2}\left(P_{2,2}, Q_{2,2}\right)$ has $x_{2}$ links. Then the $k$ th link of $C\left(1 \leqq k \leqq x_{2}\right)$ is the sum of all links of $C^{\prime}\left(P_{1,2}, Q_{1,2}\right)$ whose intersection with $H_{1,2}$ is mapped by $T$ into the $k$ th link of $D_{2}\left(P_{2,2}, Q_{2,2}\right)$. If $D_{2}\left(P_{2,3}, Q_{2,3}\right)$ has $x_{3}$ links, the last $x_{3}$ links of $C$ are defined in a similar fashion. $C^{\prime}\left(Q_{1,2}, P_{1,3}\right)$ is consolidated in such a way that $C\left(Q_{1,2}, P_{1,3}\right)$ has the same number of links as $D_{2}\left(Q_{2,2}, P_{2,3}\right)$.

We now proceed exactly as in the proof of Theorem 4 until we reach Step 5. Then we require that the chain $F$ constructed there have mesh less than $\delta$ and such that the number of links in $F\left(Q_{1,1}, X\right)$ plus the number of links in $C$ be at least as great as the number of links in $D_{2}\left(Q_{2,1}, P_{2,2}\right)$. 
Step 6 is also modified as follows. If $D_{2}\left(P_{2,1}, Q_{2,1}\right)$ has $x_{1}$ links, the $k$ th link of $D_{1}$ is the sum of all links of $F\left(P_{1,1}, Q_{1,1}\right)$ whose intersection with $H_{1,1}$ is mapped by $T$ into the $k$ th link of $D_{2}\left(P_{2,1}, Q_{2,1}\right)\left(k=1,2, \cdots, x_{1}\right) . F\left(Q_{1,2}, X\right)$ is consolidated so that $D_{1}\left(Q_{1,1}, P_{1,2}\right)$ has the same number of links as $D_{2}\left(Q_{2,1}, P_{2,2}\right)$ and $F\left(X, Q_{1,3}\right)$ is consolidated according to the same scheme used in the proof of Theorem 4 . The chain $D_{1}$ arrived at in this way is the one required.

Now suppose that this lemma is true for all integers less than $n$. The proof that the lemma is true for the integer $n$ will be very similar to the proof of Theorem 4 for the corresponding case. And as before we shall only indicate the necessary modifications in the constructions that are needed to obtain chains satisfying the additional requirements.

Corresponding to Step $1^{\prime}$ of the proof of Theorem 4, we find chains $C_{1}, C_{2}$ satisfying the conclusions of the lemma relative to $H_{1,2}, H_{1,3}, \cdots, H_{1, n}$ and $H_{2,2}, H_{2,3}, \cdots, H_{2, n}$ respectively.

Then we perform Steps $2^{\prime}-6^{\prime}$ exactly as in the proof of Theorem 4 to obtain a chain $D_{2}$ satisfying the conclusions of the lemma.

To obtain $D_{1}$ we modify these steps in a fashion similar to the way Steps 5 and 6 for the case $n=3$ were modified. In particular, Steps $2^{\prime}$ and $4^{\prime}$ remain unaltered while Step $5^{\prime}$ requires the following adjustment. 'The chain $F$ used there is now required to satisfy the added condition that the number of links of $F\left(Q_{1,1}, X\right)$ plus the number of links of $C_{1}$ be at least as great as the number of links in $D_{2}\left(Q_{2,1}, P_{2,2}\right)$ and also $\mu(F)$ must be less than $\delta$.

Step $6^{\prime}$ is modified in the same way that Step 6 for the case $n=3$ was altered. More precisely, if $D_{2}\left(P_{\llcorner, 1}, Q_{2,1}\right)$ has $x_{1}$ links, the $k$ th link of $D_{1}$ is the sum of all the links of $F\left(P_{1,1}, Q_{1,1}\right)$ whose intersection with $H_{1,1}$ is mapped by $T$ into the $k$ th link of $D_{2}\left(P_{2,1}, Q_{2,1}\right)\left(k=1,2, \cdots, x_{1}\right)$ and $F\left(Q_{1,2}, X\right)$ is consolidated so that $D_{1}\left(Q_{1,1}, P_{1,2}\right)$ has the same number of links as $D_{2}\left(Q_{2,1}, P_{2,2}\right)$. $F\left(X, Q_{1,3}\right)$ is consolidated in the same way as in Theorem 4 . The chains $D_{1}$ $D_{2}$ arrived at in this way satisfy the conclusions of the lemma.

Proof of Theorem 6. Without loss of generality we assume that $T\left(H_{1, j}\right)$ $=H_{2, j}(j=1,2, \cdots, n)$. Suppose that for some $j, H_{1, j}$ (and $\left.H_{2, j}\right)$ is degenerate. Let $H_{1, j}^{\prime}, H_{2, j}^{\prime}$ be nondegenerate, proper subcontinua of $M$ that contain $H_{1, j}, H_{2, j}$ respectively. By Theorem $2, H_{1, j}^{\prime}$ and $H_{2, j}^{\prime}$ are each pseudo-arcs and homeomorphic to one another. Then since the pseudo-arc is homogeneous, there exists an extension of $T$ taking $H_{1,1}+\cdots+H_{1, j}^{\prime}+\cdots+H_{1, n}$ onto $H_{2,1}+\cdots+H_{2, j}^{\prime}+\cdots+H_{2, n}$. Hence without loss of generality, we now assume that no $H_{1, j}$ is degenerate.

Let $P_{1, j}, Q_{1, j}$ be points that lie in different composants of $H_{1, j}(j=1,2, \cdots$, $n)$. Let $T\left(P_{1, j}\right), T\left(Q_{1, j}\right)$ be denoted by $P_{2, j}, Q_{2, j}$ respectively and note that $P_{2, j}, Q_{2, j}$ lie in different composants of $H_{2, j}$. Let $\mathcal{F}_{i}=H_{i, 1}+H_{i, 2}+\cdots$ $+H_{i, n}(i=1,2)$.

We shall also assume that all the chains that appear in the proof of this 
theorem will be consolidations of chains of the given sequence $W_{1}, W_{2}, \cdots$.

Now the proof will proceed by an induction on $n$. We consider first the case $n=3$. (If $n=1$ or 2 , we can enlarge $\mathcal{H}_{i}$, so that the case $n=3$ applies.) We divide the proof into 10 steps so as to make more transparent the proof for the case of arbitrary $n$. The basic idea will be to construct sequences of chains $D_{i, 1}, D_{i, 2}, \cdots$ which by Theorem 5 , will induce the desired extension of $T$. To do this, a rather large number of auxiliary chains will have to be constructed.

Step 1. By Lemma 4, there exist chains $E_{1,1}$ and $D_{2,1}$ each of which covers $M$ and such that (1) $E_{1,1}$ is a chain from $P_{1,1}$ to $Q_{1,3},(2) \mu\left(E_{1,1}\right)$ is less than $1 / 4$, (3) $E_{1,1}$ has property $S$ relative to $H_{1,1}, P_{1,1}, Q_{1,1}, \cdots, H_{1,3}, P_{1,3}, Q_{1,3},\left(1^{\prime}\right) D_{2,1}$ is a chain from $P_{2,1}$ to $Q_{2,3},\left(2^{\prime}\right) D_{2,1}$ has property $S$ relative to $H_{2,1}, P_{2,1}$, $Q_{2,1}, \cdots, H_{2,3}, P_{2,3},\left(3^{\prime}\right) D_{2,1}\left(P_{2, j}, Q_{2, j}\right)$ has the same number of links as $E_{1,1}\left(P_{1, j}, Q_{1, j}\right)(j=1,2,3),\left(4^{\prime}\right) D_{2,1}\left(Q_{2, j}, P_{2, j+1}\right)$ has the same number of links as $E_{1,1}\left(Q_{1, j}, P_{1, j+1}\right)(j=1,2)$, and $\left(5^{\prime}\right)$ if $d(2,1)_{k}$ is the $k$ th link of $D_{2,1}$ and intersects $\mathcal{F C}_{2}$, then $T^{-1}\left[d(2,1)_{k} \cap \mathcal{F}_{2}\right]$ is contained in the $k$ th link of $E_{1,1}$. (Note that $3^{\prime}$ and $4^{\prime}$ together imply that $D_{2,1}$ has the same number of links as $E_{1,1}$.)

Step 2. Let $D_{2,1}^{\prime}$ be a $V$-chain of $D_{z, 1}$ relative to $H_{2,1}, P_{2,1}, Q_{2,1}, \cdots$, $H_{2,3}, P_{2,3}, Q_{2,3}$. Let $E_{2,2}$ be a chain from $P_{2,1}$ to $Q_{2,3}$ that covers $M$ and such that (1) $E_{2,2}$ is contained in $D_{2,1}^{\prime}$, (2) any link of $D_{2,1}^{\prime}$ that contains a link of $E_{2,2}$ also contains the closure of that link, (3) $\mu\left(E_{2,2}\right)$ is less than $1 / 4$, (4) no link of $E_{2,2}$ intersects three links of $D_{2,1}$, and (5) $E_{2,2}$ has property $S$ relative to $H_{2,1}, P_{2,1}, Q_{2,1}, \cdots, H_{2,3}, P_{2,3}, Q_{2,3}$. (The existence of such a chain follows from Theorem 4.) Let $D_{2,2}^{\prime}$ be a chain from $P_{2,1}$ to $Q_{2,3}$ that covers $M$ and is a $V$-chain of $E_{2,2}$ relative to $H_{2,1}, P_{2,1}, Q_{2,1}, \cdots, H_{2,3}, P_{2,3}, Q_{2,3}$.

Step 3. Since $E_{1,1}$ has property $S$ relative to $H_{1,1}, P_{1,1}, Q_{1,1}, \cdots, H_{1,3}$, $P_{1,3}, Q_{1,3}$, there exists a chain $F_{1,1}$ from $P_{1,1}$ to $Q_{1,3}$ that covers $M$ and such that (1) $F_{1,1}$ is a $V$-chain of $E_{1,1}$ relative to $H_{1,1}, P_{1,1}, Q_{1,1}, \cdots, H_{1,3}, P_{1,3}, Q_{1,3}$, (2) if $f$ is any link of $F_{1,1}$ that intersects $\mathcal{F}_{1}$, then $T\left[f \cap \mathcal{F C}_{1}\right]$ is contained in a link of $D_{2,2}^{\prime}$, (3) the sum of no three consecutive links of $F_{1,1}$ intersects three links of $E_{1,1},(4) \mu\left(F_{1,1}\right)$ is less than $1 / 8$, (5) any link of $E_{1,1}$ that contains a link of $F_{1,1}$ also contains the closure of that link.

We now consolidate $F_{1,1}$ and obtain a chain $E_{1,2}^{\prime}$ as follows. If $d^{\prime}(2,2)$ is a link of $D_{2,2}^{\prime}\left(P_{2, j}, Q_{2, j}\right)(j=1,2,3)$, then the sum of the links $f$ of $F_{1,1}\left(P_{1, j}, Q_{1, j}\right)$ such that $T\left[f \cap \mathcal{F}_{1}\right]$ is contained in $d^{\prime}(2,2)$ is a link of $E_{1,2}^{\prime}\left(P_{1, j}, Q_{1, j}\right)$. The remaining links of $E_{1,2}^{\prime}$ are simply the remaining links of $F$. Since the intersection of a link $f$ of $F_{1,1}$ with $\mathcal{F C}_{1}$ may be contained in a link of $E_{1,1}$ that does not contain all of $f$ itself, $E_{1,2}^{\prime}$ may not be contained in $E_{1,1}$. Therefore in the next step we define a chain $D_{1,1}$ that does contain $E_{1,2}^{\prime}$ and which differs from $E_{1,1}$ only slightly.

Step 4. The $k$ th link of $D_{1,1}$ is the $k$ th link of $E_{1,1}$ plus any links $e^{\prime}(1,2)$ of $E_{1,2}^{\prime}$ such that $e^{\prime}(1,2) \cap \mathcal{H}_{1}$ is contained in the $k$ th link of $E_{1,1}$. Note that $D_{1,1}$ is a chain from $P_{1,1}$ to $Q_{1,3}$ that covers $M$ and whose mesh is less than 
1/2. Furthermore, since the links of $D_{1,1}, E_{1,2}^{\prime}$ are identical to those of $E_{1,1}$, $F_{1,1}$ respectively, except (perhaps) for those that intersect $\mathcal{F}_{1}, D_{1,1}$ has property $S$ relative to $H_{1,1}, P_{1,1}, Q_{1,1}, \cdots, H_{1,3}, P_{1,3}, Q_{1,3}$ and $E_{1,2}^{\prime}$ is a $V$-chain of $D_{1,1}$ relative to the same collection. In addition, if $e^{\prime}(1,2)_{k}$ is the $k$ th link of $E_{1,2}^{\prime}\left(P_{1, j}, Q_{1, j}\right)$ ( $k$ arbitrary), $T$ maps the intersection of $e^{\prime}(1,2)_{k}$ with $\mathfrak{H}_{1}$ into the $k$ th link of $D_{2,2}^{\prime}\left(P_{2, j}, Q_{2, j}\right)(j=1,2,3)$. Hence, $E_{1,2}^{\prime}\left(P_{1, j}, Q_{1, j}\right)$ follows a pattern in $D_{1,1}$ that $D_{2,2}^{\prime}\left(P_{2, j}, Q_{2, j}\right)$ follows in $D_{2,1}(j=1,2,3)$. Finally, we note that $D_{1,1}$ has the same number of links as $D_{2,1}$ and $P_{1, j}, Q_{1, j}$ are contained in the links of $D_{1,1}$ that correspond to those of $D_{2,1}$ that contain $P_{2, j}, Q_{2, j}$ respectively $(j=1,2,3)$.

In Step 5 we describe more explicitly a pattern that $D_{2,2}\left(P_{2,1}, P_{2,2}\right)$ follows in $D_{2,1}$ and in Step 6 we construct a chain $E_{1,2}^{\prime \prime}$ from $P_{1,1}$ to $Q_{1,3}$ that we shall use (Step 7) to construct a chain $D_{1,2}^{\prime}\left(P_{1,1}, P_{1,2}\right)$ from $P_{1,1}$ to $P_{1,2}$ that follows a pattern in $D_{1,1}$ that $D_{2,2}^{\prime}\left(P_{2,1}, P_{2,2}\right)$ follows in $D_{2,1}$.

Step 5. Suppose that $D_{2,1}$ has $s$ links and that $P_{2,2}$ lies in the $r$ th link of $D_{2,1}$. Since $D_{2,1}^{\prime}$ follows a $V$-pattern in $D_{2,1}$ relative to $H_{2,1}, P_{2,1}, Q_{2,1}, \cdots$, $H_{2,3}, P_{2,3}, Q_{2,3}, D_{2,1}^{\prime}$ is the sum of four subchains $D_{2,1}^{\prime}(1, t), D_{2,1}^{\prime}(t, u), D_{2,1}^{\prime}(u, v)$, $D_{2,1}^{\prime}(v, w)$ where $D_{2,1}^{\prime}(1, t)$ follows a $v$-pattern to $P_{2,2}$ in $D_{2,1}, D_{2,1}^{\prime}(t, u)$ covers $H_{2,2}$ and is contained in $D_{2,1}\left(P_{2,2}, Q_{2,2}\right)$ while $d^{\prime}(2,1)_{t}$ and $d^{\prime}(2,1)_{u}$ are each contained only in $d(2,1)_{P_{2,2}}, d^{\prime}(2,1)_{v}$ is contained only in $d(2,1)_{1}$, and $D_{2,1}^{\prime}(v, u)$ follows a $v$-pattern to $X_{2}$ in $D_{2,1}$ where $X_{2}$ is a point that is contained only in $d^{\prime}(2,1)_{u}$.

Since $D_{2,1}^{\prime}(1, t)$ follows a $v$-pattern to $P_{2,2}$ in $D_{2,1}, D_{2,1}^{\prime}(1, t)$ is the sum of $2(s-r)$ subchains $D_{2,1}^{\prime}\left(x_{2, m}, x_{2, m+1}\right)(m=1,2, \cdots, 2(s-r))$ where $(1) x_{2,1}=1$, $x_{2,2(s-r)+1}=t$, (2) $d^{\prime}(2,1)_{x_{2,2 m+1}}$ is contained only in the $(2 r-s-m)$ th link of $D_{2,1}(m=1,2, \cdots, s-r),(3) d^{\prime}(2,1)_{x_{2,2} m}$ is contained only in the $s-(m-1)$ st link of $D_{2,1}(m=1,2, \cdots, s-r)$, and (4) $D_{2,1}^{\prime}\left(x_{2,2 m}, x_{2,2 m+1}\right)$ is contained in $D_{2,1}(s-(m-1), 2 r-s+m)(m=1,2, \cdots, s-r)$.

Similarly, $D_{2,1}^{\prime}(v, u)$ is the sum of $2(s-r)$ subchains

$$
D_{2,1}^{\prime}\left(y_{2, m}, y_{2, m+1}\right) \quad(m=1,2, \cdots, 2(s-r))
$$

where (1) $y_{2,1}=v, y_{2,2(s-r)+1}=u,(2) d^{\prime}(2,1)_{y_{2,2 m+1}}$ is contained only in the $(2 r-s+m)$ th link of $D_{2,1}(m=1,2, \cdots, s-r)$, (3) $d^{\prime}(2,1)_{y_{2,2}}$ is contained only in the $s-(m-1)$ st link of $D_{2,1}(m=1,2, \cdots, s-r)$, and (4) $D_{2,1}^{\prime}\left(y_{2,2 m}, y_{2,2 m+1}\right)$ is contained in $D_{2,1}(s-(m-1), 2 r-s+m)(m=1,2$, .. , s-r).

Now let $z_{2, m}$ be the last link of $D_{2,2}^{\prime}\left(P_{2,1}, P_{2,2}\right)$ to be contained in $x_{2, m}$ $+y_{2, m}(m=2,3, \cdots, 2(s-r))$. Let $z_{2,1}$ be the first link of $D_{2,2}^{\prime}\left(P_{2,1}, P_{2,2}\right)$ and $z_{2,2(s-r) \not \equiv 1}$ the last. Then $D_{2,2}^{\prime}\left(P_{2,1}, P_{2,2}\right)$ is the sum of $2(s-r)$ subchains $D_{2,2}^{\prime}\left(z_{2, m}, z_{2, m+1}\right)(m=1,2, \cdots, 2(s-r))$ where $(1) z_{2,1}, z_{2,2(s-r)+1}$ are the first and last links of $D_{2,2}^{\prime}\left(P_{2,1}, P_{2,2}\right)$ respectively, $(2) d^{\prime}(2,2)_{z_{2,2 m+1}}$ is contained only in the $(2 r-s+m)$ th link of $D_{2,1}(m=1,2, \cdots, s-r),(3) d^{\prime}(2,2) z_{2,2 m}$ is contained only in the $s-(m-1)$ st link of $D_{2,1}(m=1,2, \cdots, s-r)$, and (4) $D_{2,2}^{\prime}\left(Z_{2,2 m}, Z_{2,2 m+1}\right)$ is contained in 


$$
D_{2,1}(s-(m-1), 2 r-s+m) \quad(m=1,2, \cdots, s-r) .
$$

Step 6. Recall (Step 4) that $E_{1,2}^{\prime}$ is a $V$-chain of $D_{1,1}$ relative to $H_{1,1}, P_{1,1}$, $Q_{1,1}, \cdots, H_{1,3}, P_{1,3}, Q_{1,3}$ such that $E_{1,2}^{\prime}\left(P_{1, j}, Q_{1, j}\right)$ follows a pattern in $D_{1,1}$ that $D_{2,2}^{\prime}\left(P_{2, j}, Q_{2, j}\right)$ follows in $D_{2,1}(j=1,2,3)$ and in addition $T$ maps the intersection of any link of $E_{1,2}^{\prime}\left(P_{1, j}, Q_{1, j}\right)$ with $\mathfrak{F}_{1}$ into the corresponding link of $D_{2,2}^{\prime}\left(P_{2, j}, Q_{2, j}\right)(j=1,2,3)$.

By using Theorem 3 , we find that there exists a chain $F_{1,2}$ and points $Y, Z$ of $M$ such that $F_{1,2}$ is a chain from $P_{1,1}$ to $Q_{1,3}$ that covers $M, F_{1,2}$ is contained in $E_{1,2}^{\prime}$, any link of $E_{1,2}^{\prime}$ that contains a link of $F_{1,2}$ also contains the closure of that link, $F_{1,2}\left(P_{1, j}, Q_{1, j}\right)$ contains $H_{j}(j=1,2,3), f(1,2)_{Y}, f(1,2)_{z}$ are contained in the first and last links respectively of $E_{1,2}^{\prime}, f(1,2)_{Q_{1,1}}<f(1,2)_{Y}$, $f(1,2)_{Z}<f(1,2)_{P_{1,3}}, F_{1,2}\left(P_{1,1}, Y\right)$ is contained in $E_{1,2}^{\prime}\left(P_{1,1}, Q_{1,1}\right), F_{1,2}\left(Z, Q_{1,3}\right)$ is contained in $E_{1,2}^{\prime}\left(P_{1,3}, Q_{1,3}\right)$, and $F_{1,2}\left(P_{1,2}, Q_{1,2}\right)$ is contained in $E_{1,2}^{\prime}\left(P_{1,2}, Q_{1,2}\right)$.

We obtain $E_{1,2}^{\prime \prime}$ by consolidating $F_{1,2}$ as follows. If $e^{\prime}(1,2)$ is a link of $E_{1,2}^{\prime}\left(P_{1,1}, Q_{1,1}\right)$ the sum of the links of $F_{1,2}\left(P_{1,1}, Q_{1,1}\right)$ contained in $e^{\prime}(1,2)$ is a link of $E_{1,2}^{\prime \prime}$. Every interior link of $F_{1,2}\left(Q_{1,1}, Y\right)$ is a link of $E_{1,2}^{\prime \prime}$. If $e^{\prime}(1,2)$ is any link of $E_{1,2}^{\prime}$, the sum of the links of $F_{1,2}(Y, Z)$ that are contained in $e^{\prime}(1,2)$ is a link of $E_{1,2}^{\prime \prime}$. Every interior link of $F_{1,2}\left(Z, P_{1,3}\right)$ is a link of $E_{1,2}^{\prime \prime}$. Finally, if $e^{\prime}(1,2)$ is a link of $E_{1,2}^{\prime}\left(P_{1,3}, Q_{1,3}\right)$, then the sum of the links of $F_{1,2}\left(P_{1,3}, Q_{1,3}\right)$ that are contained in $e^{\prime}(1,2)$ is a link of $E_{1,2}^{\prime \prime}$.

The chain $E_{1,2}^{\prime \prime}$ arrived at in this way has the following properties: (1) $E_{1,2}^{\prime \prime}$ is a chain from $P_{1,1}$ to $Q_{1,3}$ that covers $M$ and is contained in $D_{1,1},(2)$ any link of $D_{1,1}$ that contains a link of $E_{1,2}^{\prime \prime}$ contains the closure of that link, (3) $E_{1,2}^{\prime \prime}\left(P_{1, j}, Q_{1, j}\right)$ covers $H_{j}$ and follows a pattern in $D_{1,1}$ that $D_{2,2}^{\prime}\left(P_{2, j}, Q_{2, j}\right)$ follows in $D_{2,1}(j=1,2,3)$, (4) $T$ maps the intersection of any link of $E_{1,2}^{\prime \prime}\left(P_{1, j}, Q_{1, j}\right)$ with $\mathfrak{F}_{1}$ into the corresponding link of $D_{2,2}^{\prime}\left(P_{2, j}, Q_{2, j}\right)(j=1,2,3)$, (5) $E_{1,2}^{\prime \prime}(Y, Z)$ follows every pattern in $D_{1,1}$ that $E_{1,2}^{\prime}$ does, and $(6) E_{1,2}^{\prime \prime}\left(P_{1,1}, Y\right)$, $E_{1,2}^{\prime \prime}\left(Z, Q_{1,3}\right)$ are contained in $D_{1,1}\left(P_{1,1}, Q_{1,1}\right), D_{1,1}\left(P_{1,3}, Q_{1,3}\right)$ respectively.

Recall (Step 4) that $D_{1,1}$ has the same number of links as $D_{2,1}$ (i.e., $s$, Step 5) and $P_{1,2}$ lies in the link of $D_{1,1}$ that corresponds to the link of $D_{2,1}$ containing $P_{2,2}$ (i.e., $r$, also Step 5). Then since $E_{1,2}^{\prime}$ is a $V$-chain of $D_{1,1}$ relative to $H_{1,1}, P_{1,1}, Q_{1,1}, \cdots, H_{1,3}, P_{1,3}, Q_{1,3}$ and $E_{1,2}^{\prime \prime}(Y, Z)$ follows every pattern in $D_{1,1}$ that $E_{1,2}^{\prime}$ does, we find (compare with Step 5) that $E_{1,2}^{\prime \prime}(Y, Z)$ is the sum of $4(s-r)+3$ subchains $E_{1,2}^{\prime \prime}\left(x_{1, m}, x_{1, m+1}\right), E_{1,2}^{\prime \prime}\left(P_{1,2}, Q_{1,2}\right), E_{1,2}^{\prime \prime}\left(Q_{1,2}, X_{1}\right)$, $E_{1,2}^{\prime \prime}\left(y_{1, m}, Y_{1, m+1}\right) \quad(m=1,2, \cdots, 2(s-r))$ and $E_{1,2}^{\prime \prime}\left(y_{1,1}, y_{1,2(s-r)+2}\right)$, where (1) $x_{1,1}=e^{\prime \prime}(1,2)_{Y}, e^{\prime \prime}(1,2)_{x_{1,2(1-r)+1}}$ is $e^{\prime \prime}(1,2)_{P_{1,2}},(2) e^{\prime \prime}(1,2)_{x_{1,2 m+1}}$ is contained only in the $(2 r-s+m)$ th link of $D_{1,1}(m+1,2, \cdots, s-r)$, (3) $e^{\prime \prime}(1,2)_{x_{1,2 m}}$ is contained only in the $s-(m-1)$ st link of $D_{1,1}(m=1,2, \cdots, s-r)$, (4) $E_{1,2}^{\prime \prime}\left(x_{1,2 m}, x_{1,2 m+1}\right)$ is contained in $D_{1,1}(s-(m-1), 2 r-s+m)(m=1,2, \cdots, s-r)$, (5) $E_{1,2}^{\prime \prime}\left(P_{1,2}, Q_{1,2}\right)$ is contained in $D_{1,1}\left(P_{1,2}, Q_{1,2}\right),(6) X_{1}$ is a point of $M$ such that $e^{\prime \prime}(1,2)_{X_{1}}$ is contained in $d(1,1)_{P_{1,2}}$ and $E_{1,2}^{\prime \prime}\left(Q_{1,2}, X_{1}\right)$ is contained in $D_{1,1}\left(P_{1,2}, Q_{1,2}\right),(7) e^{\prime \prime}(1,2)_{y_{1,1}}$ is 
contained in $d(1,1)_{1},(8) e^{\prime \prime}(1,2)_{X_{1}}$ is $e^{\prime \prime}(1,2)_{y_{1,2(\theta-r)+1}},(9) e^{\prime \prime}(1,2)_{y_{1,2 m+1}}$ is contained only in the $(2 r-s+m)$ th link of $D_{1,1}(m=1,2, \cdots, s-r)$, (10) $e^{\prime \prime}(1,2)_{y_{1,2}}$ is contained only in the $s-(m-1)$ st link of $D_{1,1}(m=1,2, \cdots, s-r),(11) E_{1,2}\left(y_{1,2 m}, y_{1,2 m+1}\right)$ is contained in $D_{1,1}(s-(m-1), 2 r-s+m)(m=1,2, \cdots, s-r)$, and $(12) e^{\prime \prime}(1,2)_{y_{1,2(t-r)+2}}$ is $e^{\prime \prime}(1,2)_{z}$.

Step 7. In this step we make repeated use of Theorem 1 in order to obtain a chain $D_{1,2}^{\prime}\left(P_{1,1}, P_{1,2}\right)$ from $P_{1,1}$ to $P_{1,2}$ that follows a pattern in $D_{1,1}$ that $D_{2,2}^{\prime}\left(P_{2,1}, P_{2,2}\right)$ follows in $D_{2,1}$.

By the corollary to Theorem 3 , there exists a sequence of chains $E_{1,2}^{\prime \prime}$ $=C_{1}, C_{2}, \cdots$ from $P_{1,1}$ to $Q_{1,3}$ such that for each positive integer $m, C_{m+1}$ is crooked in $C_{m}$, any link of $C_{m}$ that contains a link of $C_{m+1}$ also contains the closure of that link, and the mesh of $C_{m}$ is less than $1 / m$.

Then by using Theorem 1, we find that there exists an integer $j_{0}$ and a chain $F_{0}$ such that $F_{0}$ is a consolidation of the links of $C_{j_{0}}$ that are contained in $E_{1,2}^{\prime \prime}\left(Y, Q_{1,1}\right) . F_{0}$ follows a pattern in $D_{1,1}\left(P_{1,1}, Q_{1,1}\right)$ that $E_{1,2}^{\prime \prime}\left(P_{1,1}, Q_{1,1}\right)$ does, and no interior link of $F_{0}$ intersects $e^{\prime \prime}(1,2)_{Y}+e^{\prime \prime}(1,2)_{Q_{1,1}}$.

Also there exists an integer $j_{1}$ and a chain $F_{1}$ such that $F_{1}$ is a consolidation of the links of $C_{j_{1}}$ that are contained in $E_{1,2}^{\prime \prime}\left(x_{1,1}, x_{1,2}\right), F_{1}$ follows a pattern in $D_{1,1}$ that $D_{2,2}^{\prime}\left(Z_{2,1}, Z_{2,2}\right)$ follows in $D_{2,1}$, and no interior link of $F_{1}$ intersects $e^{\prime \prime}(1,2)_{x_{1,1}}+e^{\prime \prime}(1,2)_{x_{1,2},}$.

Similarly, there exists integers $j_{2}, j_{3}, \cdots, j_{2(s-r)}$ and chains $F_{2}, F_{3}, \cdots$, $F_{2(8-r)}$ such that $F_{m}$ is a consolidation of the links of $C_{j_{m}}$ that are contained in $E_{1,2}^{\prime \prime}\left(x_{1, m}, x_{1, m+1}\right), F_{m}$ follows a pattern in $D_{1,1}$ that $D_{2,2}^{\prime}\left(Z_{2, m}, Z_{2, m+1}\right)$ follows in $D_{2,1}$, and no interior link of $F_{m}$ intersects

$$
e^{\prime \prime}(1,2)_{x_{1, m}}+e^{\prime \prime}(1,2) x_{1, m+1} \quad(m=2,3, \cdots,(s-r)) .
$$

Let $j$ be an integer at least as great as the maximum of $j_{0}, j_{1}, \cdots, j_{2(s-r)}$. Then by using the chains $F_{0}, F_{1}, \cdots, F_{2(s-r)}$ found above, we obtain a chain $D_{1,2}^{\prime}\left(P_{1,1}, P_{1,2}\right)$ from $P_{1,1}$ to $P_{1,2}$ that is a consolidation of the links of $C_{j}$ that are contained in $E_{1,2}^{\prime \prime}\left(P_{1,1}, P_{1,2}\right)$ and such that $(1) D_{1,2}^{\prime}\left(P_{1,1}, P_{1,2}\right)$ follows a pattern in $D_{1,1}$ that $D_{2,2}^{\prime}\left(P_{2,1}, P_{2,2}\right)$ follows in $D_{2,1}$, and (2) if $d^{\prime}(1,2)_{k}$ is a link of $D_{1,2}^{\prime}\left(P_{1,1}, Q_{1,1}\right), T$ maps the intersection of this link with $\mathcal{F}_{1}$ into the $k$ th link of $D_{2,2}^{\prime}\left(P_{2,1}, Q_{2,2}\right)$.

Step 8. By following a procedure similar to that gone through above, we finally obtain a chain $D_{1,2}^{\prime}$ from $P_{1,1}$ to $Q_{1,3}$ that covers $M$ and such that (1) $D_{1,2}^{\prime}$ follows a pattern in $D_{1,1}$ that $D_{2,2}^{\prime}$ follows in $D_{2,1},(2) D_{1,2}^{\prime}\left(P_{1, j}, Q_{1, j}\right)$ covers $H_{j}(j=1,2,3)$, and $(3)$ if $d^{\prime}(1,2)_{k}$ is a link of $D_{1,2}^{\prime}\left(P_{1, j}, Q_{1, j}\right), T$ maps the intersection of $d^{\prime}(1,2)_{k}$ with $H_{j}$ into the corresponding link of $D_{2,2}^{\prime}\left(P_{2, j}, Q_{2, j}\right)(j=1,2,3)$.

Step 9 . In this step we consolidate $D_{1,2}^{\prime}$ and obtain a chain $D_{1,2}$ that will correspond to the chain $E_{2,2}$. 
If the $i$ th link of $D_{2,2}^{\prime}$ is contained in the $j$ th link of $E_{2,2}$, let the $j$ th link of $D_{1,2}$ contain the $i$ th link of $D_{1,2}^{\prime}$. Though $D_{1,2}$ may not be contained in $D_{1,1}$, this much is true. If the $i$ th link of $D_{2,2}^{\prime}$ is also contained in the $k$ th link of $D_{2,1}$, then the $j$ th link of $D_{1,2}$ is contained in the sum of the $k$ th link of $D_{1,1}$ and one of the adjacent links.

Thus, if the $j$ th link of $E_{2,2}$ intersects the $k$ th link of $D_{2,1}$, the distance between the $j$ th link of $D_{1,2}$ and the $k$ th link of $D_{1,1}$ is less than $\mu\left(D_{1,1}\right)$ and if the $j$ th link of $D_{1,2}$ intersects the $k$ th link of $D_{1,1}$ the distance between the $j$ th link of $E_{2,2}$ and the $k$ th link of $D_{2,1}$ is less than $\mu\left(D_{2,1}\right)$.

Note also that if a link of $D_{1,2}$ intersects $\mathfrak{H}_{1}, T$ maps the intersection of this link with $\mathcal{H}_{1}$ into the corresponding link of $E_{2,2}$.

Since $D_{2,2}^{\prime}$ follows a $V$-pattern in $E_{2,2}$ relative to $H_{2,1}, P_{2,1}, Q_{2,1}, \cdots$, $H_{2,3}, P_{2,3}, Q_{2,3}, D_{1,2}$ follows a $V$-pattern in $D_{1,2}$ relative to $H_{1,1}, P_{1,1}, Q_{1,1}, \cdots$, $H_{1,3}, P_{1,3}, Q_{1,3}$ and $D_{1,2}$ has property $S$ relative to the same collection.

Step 10. In this step we indicate how this process may be continued so as to obtain the chains $D_{i, 1}, D_{i, 2}, \cdots(i=1,2)$ that we shall use in defining the desired extension of $T$.

In general, we proceed exactly as before (Steps 1-9) with one added precaution. Recall that each link $d(2,2)$ of $D_{2,2}$ (Step 4) will be the sum of a link $e(2,2)$ of $E_{2,2}$ plus (perhaps) some links $f(2,2)$ of $F_{2,2}$ that intersect $e(2,2)$. In obtaining $F_{2,2}$ then we require that the mesh of $F_{2,2}$ be less than $1 / 8$ and in addition also so small that if $e(2,2)$ and $d(2,1)$ are any two links of $E_{2,2}, D_{2,1}$ respectively that are a positive distance apart, then $(1) d(2,2)$ (the link of $D_{2,2}$ containing $e(2,2)$ ) is also at a positive distance from $d(2,1)$, and $(2) d(2,2)$ is contained in every link of $D_{2,1}$ that contained $e(2,2)$. With this added precaution, the following statements are true: (1) $\mu\left(D_{2,2}\right)<1 / 2$, (2) $D_{2,2}$ and $D_{1,2}$ have the same number of links, (3) if the $j$ th link of $D_{2,2}$ intersects the $k$ th link of $D_{2,1}$, the distance between the $j$ th link of $D_{1,2}$ and the $k$ th link of $D_{1,1}$ is less than $2 \mu\left(D_{1,1}\right)$, (4) if the $j$ th link of $D_{1,2}$ intersects the $k$ th link of $D_{1,1}$, the distance between the $j$ th link of $D_{2,2}$ and the $k$ th link of $D_{2,1}$ is less than $\mu\left(D_{2,1}\right)$ and (5) if a link of $D_{1,2}$ intersects $\mathcal{H C}_{1}, T$ maps the intersection of this link with $\mathcal{F}_{1}$ into the corresponding link of $E_{2,2}$.

In general then we obtain chains $D_{2,1}, D_{1,1}, D_{1,2}, D_{2,2}, D_{1,3}, \cdots$ such that (1) $D_{i, j}$ is a chain from $P_{i, 1}$ to $Q_{i, 3}$ that covers $M(i=1,2, j=1,2, \cdots)$,

$$
\begin{aligned}
\mu\left(D_{1,2 j-1}\right)<\frac{1}{2^{j}}, \quad \mu\left(D_{1,2 j}\right)<\frac{1}{2^{j-1}}, \quad \mu\left(D_{2,2 j}\right)<\frac{1}{2^{j}}, \quad \mu\left(D_{2,2 j+1}\right)<\frac{1}{2^{j-1}}, \\
(j=1,2, \cdots),
\end{aligned}
$$

(3) $D_{1, j}$ and $D_{2, j}$ each have the same number of links, (4) if the $k$ th link of $D_{i, j+1}$ intersects the $m$ th link of $D_{i, j}$, the distance between the $k$ th link of $D_{i^{\prime}, j+1}$ and the $m$ th link of $D_{i^{\prime}, j}$ is less than $2 \mu\left(D_{i^{\prime}, j}\right)\left(i, i^{\prime}=1,2, j=1,2, \cdots\right)$, 
and (5) if the $k$ th link of $D_{1,2 j}$ intersects $\mathfrak{H C}_{1}, T$ maps their common part into the $k$ th link of $D_{2,2 j}(j=1,2, \cdots)$, (6) if the $k$ th link of $D_{2,2 j-1}$ intersects $\mathfrak{K}_{2}, T^{-1}$ maps their common part into the $k$ th link of $D_{1,2 j-1}(j=1,2, \cdots)$.

Then Theorem 5 applies to this sequence of chains and the homeomorphism of $M$ onto $M$ induced by them is the desired extension of $T$.

The proof for the case of $n$ an arbitrary positive integer is almost identical to the proof for the case $n=3$. In fact, the only changes necessary in Steps 1-7 are purely notational. Step 8 is complicated only to the extent that some extra applications of Theorem 1 are necessary to obtain the chain $D_{1,2}$. And the changes in Steps 9 and 10 are again only changes in notation. As an easy consequence we have

Proof of Theorem 7. The necessity of the condition is obvious.

In proving sufficiency, we first consider the case that $M$ is irreducible between each pair of components of $\mathfrak{H}_{1}$. Then the condition stated implies that $M$ is irreducible between each pair of components of $\mathfrak{H}_{2}$ and the desired extension exists by Theorem 6 .

In case $M$ is not irreducible between each pair of components of $\mathcal{H C}_{1}$, various possibilities arise. We will consider one of these cases in detail. In particular, suppose that $M$ contains a proper subcontinuum $A$ containing $H_{1,1}$, $H_{1,2}$, and $H_{1,3}$ and that $M$ is irreducible between each pair of the sets $A$, $H_{1,4}, \cdots, H_{1, n}$. Suppose further that $A$ contains a proper subcontinuum $B$ that is irreducible about $H_{1,1}$ and $H_{1,2}$ and that $A$ is irreducible from $B$ to $H_{1,3}$. Then by choosing points $P_{1}, P_{2}, P_{3}$ in $H_{1,1}, H_{1,2}, H_{1,3}$ respectively, we see that the hypothesized homeomorphism of $M$ onto $M$ taking $P_{i}$ onto $T\left(P_{i}\right)(i=1,2,3)$ implies the existence of a proper subcontinuum $A^{\prime}$ of $M$ containing $H_{2,1}, H_{2,2}$, and $H_{2,3}$. In addition we note that $A^{\prime}$ contains a proper subcontinuum $B^{\prime}$ that is irreducible about $H_{2,1}$ and $H_{2,2}$ and that $A^{\prime}$ is irreducible from $B^{\prime}$ to $H_{2,3}$. In addition the existence of a homeomorphism of $M$ onto $M$ that agrees with $T$ on a set of points $P_{1}, P_{4}, \cdots, P_{n}$ (where $P_{i}$ is arbitrarily chosen in $I I_{1, i}$ ) shows that $M$ is irreducible between each pair of the sets $A^{\prime}, H_{2,4}, \cdots, H_{2, n}$. Then by Theorem 6 there exists a homeomorphism $T_{1}$ of $B$ onto $B^{\prime}$ that agrees with $T$ on $H_{1,1}$ and $H_{1,2}$. Again by Theorem 6, there exists a homeomorphism $T_{2}$ of $A$ onto $A^{\prime}$ that agrees with $T_{1}$ on $B$ and with $T$ on $H_{1,3}$. A final application of Theorem 6 yields a homeomorphism of $M$ onto $M$ that agrees with $T_{2}$ on $A$ and with $T$ on $H_{1,4}, H_{1,5}, \cdots, H_{1, n}$. This homeomorphism is the desired extension of $T$.

The remaining possibilities are disposed of by an extension of the techniques used above.

\section{BIBLIOGRAPHY}

1. R. H. Bing, $A$ homogeneous indecomposable plane continuum, Duke Math. J. vol. 15 (1948) pp. 729-742. 
2. G. Choquet, Prolongements d'homéomorphies. Ensembles topologiquement nommables. Caracterisation topologique individuelle des ensembles fermés totalement discontinus, C. R. Acad. Sci. Paris vol. 219 (1944) pp. 542-544.

3. H. J. Cohen, Some results concerning homogeneous plane continua, Duke Math. J. vol. 18 (1951) pp. 467-474.

4. B. Knaster and C. Kuratowski, Problème 2, Fund. Math. vol. 1 (1920) p. 223.

5. S. Mazurkiewicz, Sur les continus homogènes, Fund. Math. vol. 5 (1924) pp. 137-146.

6. E. E. Moise, An indecomposable plane continuum which is homeomorphic to each of its nondegenerate subcontinua, Trans. Amer. Math. Soc. vol. 63 (1948) pp. 581-594.

7. W. Sierpinski, Sur une propriete topologique des ensembles denombrables denses en soi, Fund. Math. vol. 1 (1920) pp. 11-16.

8. Z. Waraskiewicz, Sur les courbes planes topologiquement homogènes, C. R. Acad. Sci. Paris vol. 204 (1937) pp. 1388-1390.

UNIVERSITY OF MARYLAND,

College Park, Maryland 